\title{
Le cadre radiométrique de la séquence solutréo- badegoulienne du Cuzoul de Vers (Lot, France) : lecture critique et compléments
}

The radiometric framework of the Solutrean and Badegoulian sequence of Le

Cuzoul-de-Vers (Lot, France): critical view and new data

Sylvain Ducasse, Jean-Marc Pétillon et Caroline Renard

\section{OpenEdition \\ Journals}

Édition électronique

URL : http://journals.openedition.org/paleo/2721

DOI : $10.4000 /$ paleo. 2721

ISSN : 2101-0420

Éditeur

SAMRA

Édition imprimée

Date de publication : 28 décembre 2014

Pagination : $37-58$

ISSN : 1145-3370

Référence électronique

Sylvain Ducasse, Jean-Marc Pétillon et Caroline Renard, « Le cadre radiométrique de la séquence solutréo-badegoulienne du Cuzoul de Vers (Lot, France) : lecture critique et compléments », PALEO [En ligne], 25 | 2014, mis en ligne le 29 juillet 2015, consulté le 07 juillet 2020. URL : http:// journals.openedition.org/paleo/2721; DOI : https://doi.org/10.4000/paleo.2721

\section{c)}

PALEO est mis à disposition selon les termes de la licence Creative Commons Attribution - Pas d'Utilisation Commerciale - Pas de Modification 4.0 International. 


\title{
Le cadre radiométrique de la séquence solutréo-badegoulienne du Cuzoul-de-Vers (Lot, France) : lecture critique et compléments
}

\author{
Sylvain DUCASSE(1), Jean-Marc PÉTILLON ${ }^{(2)}$,Caroline RENARD(2)
}

\begin{abstract}
Résumé : Publiée en fin d'année 2012, la monographie du gisement solutréen et badegoulien du Cuzoul-de-Vers (Lot) présente l'état d'une recherche pluridisciplinaire menée sur près de 15 ans. Elle rend disponible les données acquises au terme des années 2000 sur l'un des sites ouest-européens les plus importants pour l'étude des sociétés du Dernier Maximum glaciaire. Dans son sillage, de véritables approches interdisciplinaires ont vu le jour, approches nourrissant des projets qui dépassent aujourd'hui le cadre de cet ouvrage. Parmi ces compléments et/ou extensions, le présent article propose une relecture critique du cadre radiométrique à la lueur d'une nouvelle série de dates ${ }^{14} \mathrm{C}$ par SMA obtenue avec la collaboration des laboratoires de Lyon, Saclay et Oxford. Résultant de la datation directe de plusieurs déchets de débitage en bois de renne badegouliens et restes fauniques solutréens dont la localisation stratigraphique a été préalablement contrôlée, les 11 nouvelles mesures nous permettent de discuter, de préciser et/ou de modifier les résultats précédemment publiés. Ces données tendent à confirmer (1) la faible amplitude chronologique de l'ensemble de la séquence du Cuzoul-de-Vers, (2) l'ancienneté des occupations badegouliennes, (3) la chronologie resserrée des niveaux solutréens et (4) la fugacité de certains « épisodes » d'occupation dont l'individualisation s'avère aujourd'hui illusoire sur le plan radiométrique. Accompagnant l'analyse archéostratigraphique aujourd'hui en cours, ce travail préliminaire illustre enfin la nécessité d'une redéfinition précise des ensembles étudiés. Fruit d'un travail collectif, cette démarche est aujourd'hui inscrite dans un programme plus large (PCR « SaM ») visant notamment une meilleure sériation temporelle des changements techno- et socio-économiques documentés dans la première moitié du DMG au nord des Pyrénées.
\end{abstract}

Mots-clés : Dernier Maximum Glaciaire, Solutréen récent, Badegoulien, Quercy, Le Cuzoul-de-Vers, datation ${ }^{14} \mathrm{C}$.

Abstract: The radiometric framework of the Solutrean and Badegoulian sequence of Le Cuzoul-de-Vers (Lot, France): critical view and new data. The pluridisciplinary research carried at Le Cuzoul-de-Vers (Lot, France) over 15 years has been published in late 2012. This publication makes available the data acquired at the end of the 2000s on one of the most important Western European sites for the study of the Last Glacial Maximum (LGM) societies. From this point new interdisciplinary research projects have been built leading to several complements and/or extents. Among them, a reassessment of the radiometric framework due to new AMS ${ }^{14} \mathrm{C}$ dates obtained with the collaboration of Lyon, Saclay and Oxford laboratories is presented here. Eleven new radiocarbon measurements resulting of the direct dating of Badegoulian antler waste products and Solutrean faunal remains whose stratigraphic position has been controlled allow us to specify and/or modify the results previously published. These data tend to confirm (1) the small chronological amplitude of the entire sequence, (2) the old age of the Badegoulian occupations, (3) the very short chronology of the Solutrean levels

(1) CNRS, Laboratoire PACEA (UMR 5199), Université de Bordeaux, Bâtiment B18, Allée Geoffroy St-Hilaire, CS 50023, FR-33615 Pessac cedex - s.ducasse@pacea.u-bordeaux1.fr

(2) CNRS, Laboratoire TRACES (UMR 5608), Maison de la Recherche, Université de Toulouse - Le Mirail, 5 Allées Antonio Machado, FR-31058 Toulouse cedex 9 - petillon@univ-tlse2.fr - caroline.renard@univ-tlse2.fr 
and (4) the transience of some occupation episodes whose radiometric individualization remains illusory. Led in parallel to an archeostratigraphic analysis in progress (inter-layers refitting), this preliminary work shows the necessity to precisely redefine the assemblages studied. As a collective work, this approach is part a wider program (Collective Research Program "SaM") aiming at a better chronological seriation of the techno- and socio-economic changes documented in the early part of the LGM north of the Pyrenees.

Key-words: Last Glacial Maximum, Recent Solutrean, Badegoulian, Quercy, Le Cuzoul-de-Vers, AMS ${ }^{14} \mathrm{C}$ chronology.

\section{Introduction}

Découvert en 1982 et fouillé jusqu'en 1986 sous la direction de Jean Clottes et Jean-Pierre Giraud, l'abri du Cuzoul-deVers (Lot) se situe au pied d'une falaise calcaire exposée plein sud, en bordure de la RN 653 qui le sépare du Lot distant de près de 30 mètres du fond de l'abri. Exploité durant 5 ans dans le cadre d'une fouille de sauvetage programmée, ce gisement a livré un enregistrement sédimentaire couvrant tout ou partie de la première moitié du Dernier Maximum glaciaire (DMG ; sensu Mix, Bard, Schneider 2001, MARGO project 2009). Sur les plans archéologique et sédimentaire, cette stratigraphie de référence se compose de trois couches attribuées au Solutréen supérieur (c.31 à 29), lesquelles sont surmontées de 27 couches dont les équipements lithiques et osseux permettent un rattachement au Badegoulien, depuis ses toutes premières expressions (Badegoulien ancien : couches 27 à 22) jusqu'à sa phase dite " classique » du point de vue historiographique (Badegoulien récent à raclettes : couches 21 à 1 ; Clottes et Giraud 2012).

En fin d'année 2012 paraissait la monographie du gisement, fruit d'un travail pluridisciplinaire étalé sur près de 15 ans (Clottes et al. dir., 2012). L'abondance et la diversité des vestiges exhumés (industrie lithique, industrie osseuse, parure, faune et microfaune, art mobilier, colorants, restes humains, etc.) ont conduit à la mise en œuvre d'analyses spécialisées qui, planifiées à partir de la fin des années 1990, répondaient aux approches alors classiquement attendues (Clottes et Giraud 2012). En motivant parfois de véritables approches interdisciplinaires, la conjugaison de ces études et leur mise en perspective ont permis de jeter les bases de réflexions nouvelles sur la trajectoire socioéconomique des sociétés connues dans la première moitié du DMG sur l'actuel territoire français (Ducasse et Renard 2012). Cela a également été l'occasion de porter un regard neuf et critique sur la documentation archéologique disponible, annonçant d'indispensables compléments d'analyses. Parmi ces compléments, la réévaluation du cadre archéo-stratigraphique semble incontournable compte tenu de la complexité du remplissage illustrée notamment par l'existence de raccords et remontages intercouches réalisés à partir des industries lithiques (Ducasse 2010) et osseuses (Le Guillou 2012), de la distribution verticale de certains vestiges humains attribuables à un même individu (Henry-Gambier et Villotte 2012) ou des relations stratigraphiques parfois ambigües entretenues par différentes structures foyères (Fourment et Giraud 2012). Précision néanmoins que ces différents indices, bien loin de traduire d'importantes perturbations post-dépositionnelles, semblent avant tout exprimer la faible signification archéologique du découpage sédimentaire réalisé à la fouille (cf. infra pour les niveaux solutréens) et imposé tant par la nature du remplissage (fortes variations latérales et verticales des faciès sédimentologiques) que par les contraintes du terrain (Clottes et Giraud 1989). Les nouveaux repères radiométriques obtenus au début des années 2000 ( ${ }^{14} \mathrm{C}$ par SMA : Oberlin et Valladas 2012) ont en outre considérablement modifié le cadre défini au cours des années 1980 (méthode classique ${ }^{3}$ : Clottes et Giraud 1989). Tout en vieillissant et resserrant la fourchette temporelle durant laquelle les groupes badegouliens sont venus occuper l'abri, ce renouvellement a introduit des recouvrements entre certaines phases culturelles et produit plusieurs inversions et/ou aberrations chronologiques.

De fait, et conjointement à un travail de critique taphonomique essentiel mais chronophage ${ }^{4}$, une révision du cadre radiométrique aujourd'hui disponible s'est

(3) Le terme " classique " est ici utilisé pour désigner la méthode de datation par comptage des bêta ainsi que toute mesure obtenue par ce biais. Cet usage permet d'éviter toute confusion avec l'expression "dates conventionnelles » qui désigne de son côté toute date non calibrée obtenue indifféremment par les méthodes classique (i.e. comptage des bêta) ou SMA (p.e. Valladas 2002).

(4) Très avancée pour ce qui concerne les niveaux solutréens (Fourment 2002 d'après les remontages réalisés par C. Renard, Fourment et Giraud 2012), l'analyse archéo-stratigraphique globale des riches et nombreux niveaux badegouliens est en cours, jusqu'ici suspendue à l'informatisation des bordereaux de fouille (travail entamé par deux d'entre nous - CR et SD - et achevé en 2013 par A. Angelin dans le cadre du Projet collectif de recherche «SaM »). 
imposée. Les principaux objectifs de ce travail étaient de (1) tester la réalité du vieillissement/resserrement introduit par les datations par SMA, (2) tenter de mieux appréhender l'amplitude et la position chronologique de chacune des grandes phases d'occupation documentées (Solutréen supérieur/Badegoulien ancien/Badegoulien récent) et, ce faisant, (3) identifier plus précisément les moments de " passage » de l'une à l'autre et/ou les éventuels hiatus dans l'occupation du site. Toute préliminaire qu'elle soit, notre contribution vient donc prolonger les résultats obtenus dans le cadre de la monographie en en proposant une lecture critique nourrie de nouvelles mesures ${ }^{14} \mathrm{C}$. Ces mesures ont été réalisées à partir d'échantillons sélectionnés selon un protocole simple mais systématique qui renforce la valeur des résultats obtenus et permet une critique ultérieure de l'usage qui en est fait (cf. infra : déchets en bois de renne déterminés sur le plan technologique et caractéristiques sur le plan chrono-culturel pour le Badegoulien; restes fauniques déterminés anatomiquement et/ou spécifiquement puis sélectionnés selon des critères de localisation strictement définis pour le Solutréen ; dans tous les cas : pièces cotées dont la position stratigraphique est contrôlée/contrôlable).

\section{Archéoséquence versus datations ${ }^{14} \mathrm{C}$ : problèmes et questions posés}

Les neuf campagnes de fouilles ont permis de suivre la stratigraphie du gisement sur plus de deux mètres d'épaisseur inégalement préservés (Clottes et Giraud 1989, 2012). La construction de la route qui longe le Lot ainsi que les aménagements postérieurs réalisés en vue d'améliorer la visibilité du trafic ont en effet tronqué la séquence à son sommet (p.e. couches 1 à 4 : seulement entre 2 et $10 \mathrm{~m}^{2}$ préservés). Par ailleurs, certains des niveaux les plus profonds, dégagés sur de plus grandes superficies (p.e. couches 13 à 31 : généralement plus de $30 \mathrm{~m}^{2}$ fouillés), sont en partie scellés par l'actuelle RN 653. Si l'ensemble des couches reconnues ne possède pas, de fait, la même valeur informative, le matériel qu'elles ont livré permet d'identifier des variations diachroniques dont le sens dépasse les éventuels problèmes de représentativité liés à ces différentes contraintes. Depuis les occupations humaines attribuables à la fin du Solutréen (op. cit. ; Renard 2008 et 2012) cette séquence exceptionnellement dilatée permet en effet d'observer la mise en place et l'évolution des nouvelles normes typo- et techno-économiques introduites par le Badegoulien au nord des Pyrénées. La mise en évidence d'une phase d'occupation contemporaine des premiers moments du Badegoulien fut l'un des attraits majeurs du gisement, le développement de cette phase au Cuzoul-de-Vers n'ayant pas d'égal à l'échelle ouesteuropéenne. Très vite, et à mesure que la fouille progressait, plusieurs datations ont été réalisées afin de préciser la position chronologique de chaque ensemble et permettre les comparaisons avec les rares stratigraphies comparables déjà datées à l'époque (Laugerie-Haute : Evin, Marien, Pachaudi 1976 ; Abri Fritsch : Evin, Marien, Pachaudi 1978).

\section{Ce que dit la méthode classique}

Deux séries de dates furent réalisées par la méthode classique, l'une en 1984 ( 2 dates pour le Badegoulien récent, 1 date pour le Badegoulien ancien) et l'autre en 1985 (2 dates pour le Badegoulien récent, 1 date pour le Badegoulien ancien et 1 date pour le Solutréen supérieur découvert en 1984), toutes par le laboratoire de Gif-surYvette (tabl. 1, $\mathrm{n}^{\circ} 1$ à 7 ; Delibrias et Guillier 1988). Compte tenu de la forte fragmentation des restes osseux et de leur faible teneur en collagène, les vestiges sélectionnés pour datation correspondaient à des lots d'esquilles (Oberlin et Valladas 2012). Couche 3 exceptée ${ }^{5}$, les résultats obtenus s'inscrivirent parfaitement dans le cadre radiométrique alors en cours de définition pour ces phases culturelles (fig. 1A). Bien plus précises que les mesures disponibles à LaugerieHaute et à l'abri Fritsch, ces données trouvèrent plus tard un écho remarquable à travers la séquence de l'abri Casserole, gisement fouillé au début des années 1990 (Detrain et al. 1993 et 1994).

Bien que très cohérentes dans leur succession, ces dates permettent avant tout de circonscrire l'amplitude chronologique globale des occupations humaines $d u$ Cuzoul-de-Vers ; elles ne fournissent théoriquement que des repères généraux, représentatifs de certains « morceaux choisis » de la stratigraphie (couches 5, 13, 20, $23 / 24$ et 30 , c'est-à-dire : sommet, cœur et base de la séquence du Badegoulien récent, sommet du Badegoulien ancien et base du Solutréen supérieur ${ }^{6}$ ). Après calibration, l'absence de recouvrement constaté entre Solutréen supérieur et Badegoulien ancien ainsi que le hiatus observé entre phases anciennes et récentes du Badegoulien (superposable à celui mis en évidence à Casserole : fig. 1A) ne peuvent donc être interprétés sur le plan archéologique : ces phénomènes de « regroupement », probablement accentués par les modalités d'échantillonnage (lots d'esquilles), résultent probablement bien plus de ces choix que d'une discontinuité réelle des occupations.

(5) La datation obtenue pour la couche 3 (Gif 6372 : $14560 \pm 130 \mathrm{BP}$; lots d'esquilles osseuses issues des sous-niveaux 3a et 3b) est jugée incohérente au regard du matériel archéologique qui lui est associé (Badegoulien récent, présence de nombreuses raclettes). II reste néanmoins impossible de dire si cette incohérence résulte (1) de la mauvaise qualité de l'échantillon daté, (2) d'une décontamination imparfaite ou bien si (3) certaines esquilles formant ce lot provenaient en fait d'un niveau plus récent, non déterminé à la fouille (les couches sommitales, résiduelles, ne sont connues que sur de très faibles superficies). Notons qu'un nouveau test par SMA au début des années 2000 n'a pu aboutir faute de collagène en quantité suffisante (Oberlin et Valladas 2012).

(6) La couche 31 n'étant « probablement que le tout début de l'ensemble 30 » (Clottes et Giraud 2012, p. 26). 


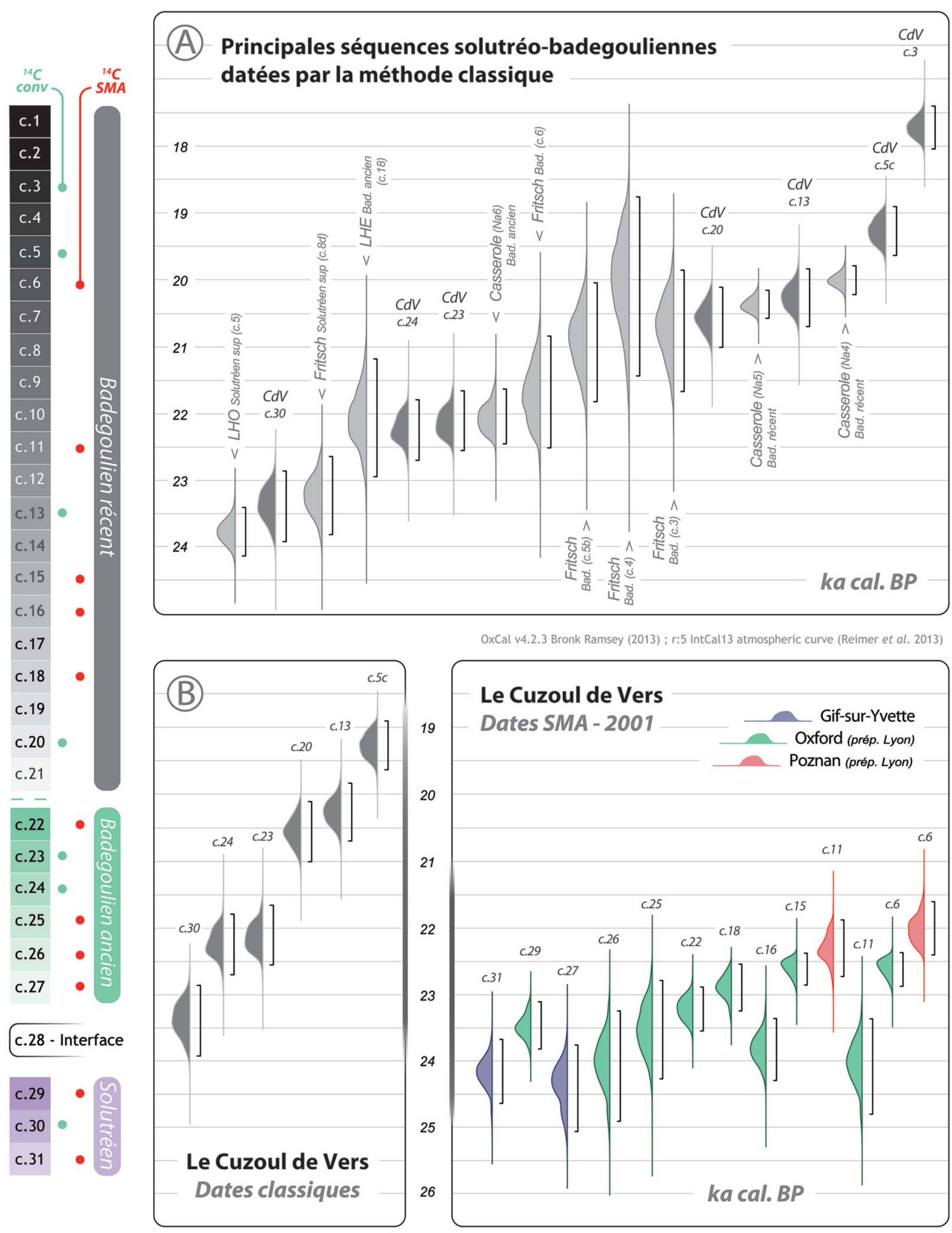

Figure 1 - Synthèse graphique de l'ensemble des datations réalisées entre 1984 et 2001 au Cuzoul-de-Vers (d'après Clottes et Giraud 1989 ; Oberlin et Valladas 2012). A : datations classiques des niveaux 3, 5, 13, 20 (Badegoulien récent), 23, 24 (Badegoulien ancien) et 30 (Solutréen supérieur) comparées aux données acquises par la méthode classique à l'abri Casserole (Detrain et al. 1993), à l'abri Fritsch et à Laugerie Haute (Evin et al. 1976 et 1978). B : Discordances entre dates classiques (à gauche) et dates par SMA (à droite) obtenues au Cuzoul-de-Vers.

Figure 1- Graphical abstract of the chronological framework established at Le Cuzoul-de-Vers between 1984 and 2001 (from Clottes and Giraud 1989; Oberlin and Valladas 2012). A: radiometric dating of layers 3, 5, 13, 20 (Recent Badegoulian), 23, 24 (Early Badegoulian) and 30 (Upper Solutrean) compared to radiometric ones from Casserole (Detrain et al. 1993), Fritsch and Laugerie Haute rock-shelters (Evin et al. 1976 and 1978). B: Discrepancies between beta counting method (on the left) and AMS method (on the right) at Le Cuzoul-de-Vers. 


\section{Ce qu'ont changé les datations par SMA}

À la fin des années 1990, la mise en route du projet de publication fut l'occasion de compléter ces données et d'affiner la temporalité des différentes phases d'occupation du site. Treize mesures supplémentaires furent ainsi réalisées, portant à 18 le nombre de couches datées (tabl. $1, \mathrm{n}^{\circ} 8$ à $20: 13$ dates pour 11 couches). In fine, en cumulant les différentes séries de dates obtenues entre le milieu des années 1980 et le début des années 2000 (Oberlin et Valladas 2012), le corpus disponible couvre une bonne part de la séquence puisqu'il concerne près de la moitié des niveaux attribués au Badegoulien récent (9 couches sur $21^{7}$ ) et la totalité de ceux rapportés au Solutréen supérieur et au Badegoulien ancien (couches 22 à 31). Bénéficiant des développements techniques liés à la spectrométrie de masse par accélérateur (SMA : Valladas et al. 2001), ces nouvelles mesures résultent théoriquement de stratégies d'échantillonnage plus précises $(1$ date $=1$ seul et même vestige $;$ pas de dates " moyennées ") et, par voie de conséquence, garantissent une meilleure précision des résultats obtenus. Les échantillons datés correspondent à des diaphyses d'ongulés de taille moyenne (non déterminées, rapportables au renne ou au bouquetin) sélectionnées par
Jean-Christophe Castel au terme de l'analyse archéozoologique (Castel 1999, 2003 et 2010). Deux échantillons ont été confiés au laboratoire de Gif-surYvette, déjà en charge des premières séries de dates (tabl. 1 : couches 27 et 31). Le laboratoire de Lyon, assurant la préparation des 11 autres pièces à dater (traitements de purification et de décontamination), a de son côté transmis les prélèvements aux laboratoires d'Oxford (9 cas) et de Poznan ( 2 cas) afin que les mesures y soient réalisées.

Les résultats obtenus ont introduit plusieurs divergences avec les dates déjà disponibles (fig. 1B). La plus évidente concerne le vieillissement du cadre radiométrique : tandis qu'à deux sigmas, et une fois les dates calibrées, le terme des occupations badegouliennes pouvait être fixé vers $19 \mathrm{ka}$ cal BP par la méthode classique (couche 5 : $15980 \pm 150$ BP, soit 19640-18910 cal. BP), les dates par SMA situent cette même limite autour de 21,5 ka cal. BP (couche $6: 18180 \pm 170 \mathrm{BP}$, soit 22417-21614 cal. BP). En outre, ces données réduisent considérablement la fourchette chronologique dans laquelle se placent les occupations du Cuzoul-de-Vers, son amplitude variant de 5000 ans $\left({ }^{14} \mathrm{C}\right.$ classique : de 24 à $\left.19 \mathrm{ka} \mathrm{BP}\right)$ à 3500 ans $\left({ }^{14} \mathrm{C}\right.$ par SMA : de 25 à 21,5 ka BP). Enfin, ce double effet

\begin{tabular}{|c|c|c|c|c|c|c|c|c|c|}
\hline & Réf. échantillon & Méthode & Couche & Attribution & Matériau & Type d'échantillon & Code laboratoire & Age BP & 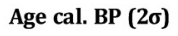 \\
\hline 1 & Carrés G2-3 / F2 & Class. & 3 & Badegoulien récent & Os & Lot d'esquilles & Gif-6372 & $14560 \pm 130$ & $18050-17409$ \\
\hline 2 & Carrés E3-4 & Class. & $5 c$ & Badegoulien récent & Os & Lot d'esquilles & Gif-6638 & $15980 \pm 150$ & 19640-18910 \\
\hline 3 & Carrés B2-4 & Class. & 13 & Badegoulien récent & Os & Lot d'esquilles & Gif-6371 & $16800 \pm 170$ & 20704-19840 \\
\hline 4 & Carrés E3-4 & Class. & 20 & Badegoulien récent & 0s & Lot d'esquilles & Gif-6797 & $17050 \pm 170$ & 21014-20115 \\
\hline 5 & ind. & Class. & 23 & Badegoulien ancien & Os & Lot d'esquilles & Gif-6370 & $18300 \pm 200$ & 22554-21663 \\
\hline 6 & ind. & Class. & 24 & Badegoulien ancien & Os & Lot d'esquilles & Gif-6798 & $18400 \pm 200$ & $22701-21796$ \\
\hline 7 & ind. & Class. & 30 & Solutréen supérieur & Os & Lot d'esquilles & Gif-6699 & $19400 \pm 210$ & $23915-22860$ \\
\hline 8 & "Vrac" & SMA & 6 & Badegoulien récent & Os & Diaphyse OTM & OxA-10955 (Lyon-1674) & $18730 \pm 110$ & $22889-22383$ \\
\hline 9 & "Vrac" & SMA & 6 & Badegoulien récent & Os & Lot de diaphyses OTM & Poz-1594 (Lyon-1962) & $18180 \pm 170$ & 22417-21614 \\
\hline 10 & Carré F2 & SMA & 11 & Badegoulien récent & Os & Diaphyse ОTM & OxA-10903 (Lyon-1675) & $19970 \pm 270$ & $24818-23380$ \\
\hline 11 & Carré F2 & SMA & 11 & Badegoulien récent & Os & Lot de diaphyses OTM & Poz-1595 (Lyon-1963) & $18480 \pm 180$ & $22742-21888$ \\
\hline 12 & Carrés B2 ou B3 & SMA & 15 & Badegoulien récent & Os & Diaphyse OTM & OxA-10974 (Lyon-1676) & $18730 \pm 100$ & $22871-22391$ \\
\hline 13 & Carré C3 & SMA & 16 & Badegoulien récent & Os & Diaphyse ОTM & OxA-10975 (Lyon-1677) & $19800 \pm 190$ & $24310-23375$ \\
\hline 14 & Carré E5 & SMA & 18 & Badegoulien récent & Os & Diaphyse OTM & OxA-11118 (Lyon-1681) & $19020 \pm 110$ & $23262-22555$ \\
\hline 15 & Carrés D2 à 5 & SMA & $22 b$ & Badegoulien ancien & Os & Diaphyse OTM & OxA-10976 (Lyon-1678) & $19280 \pm 120$ & 23563-22901 \\
\hline 16 & Carré D3 & SMA & 25 & Badegoulien ancien & Os & Diaphyse OTM & OxA-11080 (Lyon-1679) & $19540 \pm 310$ & $24285-22802$ \\
\hline 17 & Carré C4 & SMA & 26 & Badegoulien ancien & Os & Diaphyse OTM & OxA-11081 (Lyon-1680) & $19950 \pm 310$ & $24926-23261$ \\
\hline 18 & Carré C5 & SMA & 27 & Badegoulien ancien & Os & Diaphyse OTM & GifA-101441 & $20230 \pm 240$ & 25081-23776 \\
\hline 19 & Carré B5 & SMA & 29 & Solutréen supérieur & Os & Diaphyse OTM & OxA-11220 (Lyon-1682) & $19510 \pm 110$ & $23836-23124$ \\
\hline 20 & Carré I4 & SMA & 31 & Solutréen supérieur & Os & Diaphyse OTM & GifA-101440 & $20110 \pm 180$ & $24655-23689$ \\
\hline
\end{tabular}

Tableau 1 - Bilan des datations classiques ( $n^{\circ} 1$ à 7) et SMA ( $n^{\circ} 8$ à 20) réalisées entre 1984 et 2001 (d’après Clottes et Giraud 1989 ; Oberlin et Valladas 2012). OTM = Ongulé de Taille Moyenne. Les informations de localisation des échantillons datés par SMA nous ont été communiquées par J.-C. Castel. L'ensemble des calibrations a été réalisé avec l'aide du logiciel OxCal (v4.2.3 : Bronk Ramsey 2013) utilisant la courbe de calibration IntCal13 (Reimer et al. 2013).

Table 1- Results of classic ( $n^{\circ} 1$ to 7 ) and AMS datings ( $n^{\circ} 8$ to 20) made between 1984 and 2001 (after Clottes and Giraud 1989; Oberlin and Valladas 2012). OTM = Medium Sized Ungulate. Informations related to the location of dated-AMS samples were provided by J.-C. Castel. Calibration has been done with the OxCal program (v4.2.3: Bronk Ramsey 2013) using the IntCal13 calibration curve (Reimer et al. 2013).

(7) Précisons par ailleurs que la plus grande part des couches non datées correspond soit à des ensembles résiduels et parfois stériles (couches 1, 2 et 4), soit à des ensembles spatialement et/ou stratigraphiquement peu développés (couches 10, 12, 14). 
de vieillissement/resserrement est complété par une disparition des hiatus induits par les deux premières séries de dates (fig. 1B, à gauche). Probablement plus conforme au rythme réel auquel les occupations se sont succédé, cette " réorganisation " s'accompagne néanmoins de nets recouvrements entre les différentes phases chronoculturelles (p.e. distinction impossible entre Solutréen supérieur et Badegoulien ancien : fig. 1B, à droite ; Oberlin et Valladas 2012, p.80) ainsi que de plusieurs inversions qui, pour certaines, demeurent problématiques même une fois les mesures calibrées (p.e. couches 11 et 16).

\section{Dates classiques versus ${ }^{14} \mathrm{C}$ par SMA : pour un usage disjoint}

Le phénomène de resserrement des dates est donc en partie dû à la conjonction du vieillissement de l'ensemble des dates badegouliennes et de la relative stabilité des données radiométriques obtenues pour le Solutréen supérieur (entre 23,9 et 22,8 ka cal. BP en ${ }^{14} \mathrm{C}$ classique et entre 24,6 et $23 \mathrm{ka}$ cal. BP en ${ }^{14} \mathrm{C}$ par SMA $\left.{ }^{8}\right)$. S'il n'est donc pas systématique, le décalage chronologique observé entre les séries de dates classiques et par SMA - qui s'effectue toujours dans le sens d'un vieillissement - pose une nouvelle fois le problème de leur comparabilité (Bryant et al. 2001 ; Geneste 2002, p. 31 ; d'Errico, Sanchez Goni, Vanhaeren 2006 : figure 5, p. 274). En théorie, ce décalage est expliqué par l'amélioration (1) des stratégies d'échantillonnage et (2) des techniques et modalités de traitement des échantillons à dater. Pour ce qui concerne le Badegoulien et ses marges chronologiques, plusieurs réévaluations récentes permettent d'illustrer la récurrence d'un tel décalage, récurrence qui doit nous interroger sur le bien-fondé de l'utilisation conjointe, sans distinction et sans discussion, des résultats issus de ces deux types de mesures (p.e. pour le Magdalénien inférieur de l'abri Gandil : Ladier 2000, p. 197-198 ; Langlais 2010 ; pour le Badegoulien du site de plein air de Lassac : Pétillon et Ducasse 2012 ; Sacchi, Brulé, Ducasse à paraître). Tenant compte de ces éléments techniques et méthodologiques ainsi que de la répétition du phénomène pour le DMG ouest-européen, nous avons pris le parti de privilégier les dates par SMA dont le corpus ne cesse par ailleurs de croître pour cet espace-temps (p.e. Chauvière et al. 2008 ; Debout et al. 2012 ; Ducasse et al. 2011 ; Ducasse et Renard dir. 2014 ; Hinguant et Biard 2013 ; Primault et al. 2007 ; Roque et al. 2001).

\section{Quelle comparabilité entre laboratoires ?}

La question de la comparabilité des résultats ${ }^{14} \mathrm{C}$ obtenus dans des laboratoires différents est parfois posée ${ }^{9}$, et ce dans la mesure où cette différence est implicitement reliée à une variabilité potentielle des pratiques (nature des prétraitements, utilisation d'échantillons étalon différents, etc. ; voir par exemple Scott 2003, Scott, Cook, Naysmith 2007 et $2010 \mathrm{a}$ et b). À l'échelle des mesures par SMA réalisées au Cuzoul-de-Vers dans le cadre de la monographie (fig. 1B, à droite), cette question semble à première vue légitime : la datation des couches 6 et 11 montre par exemple une discordance manifeste entre les résultats obtenus à Poznan (plus récents) et Oxford (plus anciens) tandis que les dates réalisées au laboratoire de Gif-sur-Yvette ont donné (1) la date la plus ancienne pour le Solutréen supérieur (couche 31) alors même que les analyses techno-économiques et taphonomiques montrent toute la cohérence de l'ensemble c.29-30-31 (Renard 2012 ; Fourment et Giraud 2012 et infra) et (2) l'une des dates badegouliennes les plus anciennes (couche 27 : $20230 \pm 140 \mathrm{BP}$, soit 25081-23776 cal. BP), qui plus est superposable à celle de la couche 31 (20110 \pm 180 $\mathrm{BP}$, soit 24655-23689 cal. BP) et antérieure à celle de la couche 29 réalisée à Oxford $(19510 \pm 110 \mathrm{BP}$, soit 2383623124cal. BP).

En réalité, plusieurs éléments permettent, dans chaque cas, d'écarter l'idée d'un problème de comparabilité entre laboratoires. En premier lieu, les deux dates obtenues à Poznan (couches 6 et 11) doivent selon nous être considérées avec prudence - voire clairement écartées compte tenu de la nature des échantillons datés qui correspondent à des lots de diaphyses (Oberlin et Valladas 2012, p. 79 $)^{10}$. Ainsi, la plus faible précision des résultats obtenus et l'effet de rajeunissement par comparaison avec les données acquises à Oxford (notamment pour la couche 6) pourraient s'expliquer d'un point de vue méthodologique, les deux mesures réalisées par le laboratoire de Poznan renvoyant, comme les premières dates classiques, à des âges moyennés. Ensuite, si le résultat obtenu par Gif-surYvette pour la couche 27 semble aberrant, le même constat peut-être formulé au sujet de la couche 26 (voire 25) dont la datation est due au laboratoire d'Oxford, et ce à partir du même type de vestige osseux récolté, qui plus est, dans une zone similaire (tabl. 1 : carrés $\mathrm{C} 4$ et $\mathrm{C} 5$ ). Dans ce cas, le vieillissement des résultats (par ailleurs peu précis : écarts-types situés entre \pm 240 et \pm 310 ) pourrait renvoyer à des perturbations stratigraphiques dont la nature et l'ampleur restent à définir. Enfin, avant de conclure à l'imprécision de la date obtenue par le laboratoire de Gif-

(8) Pour comparaison, la base de la couche 5 est datée entre 19,5 et 18,8 ka cal. BP en ${ }^{14} \mathrm{C}$ classique tandis que la couche 6 se place entre 22,2 et 21,3 ka cal. BP en ${ }^{14} \mathrm{C}$ par SMA.

(9) Le cas de mesures ${ }^{14} \mathrm{C}$ divergentes pour un même objet daté dans deux laboratoires différents est connu : voir par exemple Alix et al. 2012 (bien que dans ce cas précis, les auteurs ne disposent pas d'élément permettant d'interpréter cet écart).

(10) Comme le rappellent les auteurs (Oberlin et Valladas 2012, p. 83), ces lots étaient originellement destinés à réaliser des mesures par la méthode classique afin de tester la réalité du vieillissement introduit par les dates obtenues par SMA. Leur faible teneur en collagène les a néanmoins contraints à opter de nouveau pour l'usage de la méthode par SMA. 
sur-Yvette pour la couche 31 (suggéré in Ducasse et Renard 2012, p. 461), il convient de garder à l'esprit que le très faible recouvrement entre ce résultat et la mesure obtenue pour la couche 29 pourrait témoigner d'un épisode d'occupation distinct : l'existence d'un niveau sous-jacent, dont la base se caractérisait par un sol «marqué par de tout petits os brûlés et quelques rares galets et éclats, trahissant un passage rapide, à une époque indéterminée, contemporaine du Solutréen ou antérieure " (couche 32 : Clottes et Giraud 2012, p. 27), ne doit pas être négligée. Tant que cette possibilité n'aura pas été écartée - et dans l'impossibilité de situer le vestige daté avec précision -, il nous semble difficile de prendre définitivement position.

\section{Quid des inversions de date?}

Une fois les deux dates réalisées à Poznan exclues, l'une des différences les plus marquantes entre datations classiques et par SMA concerne l'apparition de mesures aberrantes à l'origine de plusieurs inversions par rapport à l'ordre de la succession stratigraphique (fig. 1B, à droite). Tandis que l'usage de la méthode classique aurait tendance à atténuer d'éventuels problèmes taphonomiques (cf. supra : dates " moyennées "), l'emploi de la méthode par SMA tend à favoriser leur mise en évidence ( 1 date $=1$ vestige). Les couches badegouliennes 11, 16, 26 et 27 ont ainsi livré des résultats incohérents qu'une calibration à 2 sigmas ne permet pas de pondérer ${ }^{11}$. Toutes situées entre 24,5 et $23,5 \mathrm{ka}$ cal. BP, soit dans le même intervalle que la date la plus ancienne obtenue pour le Solutréen supérieur, ces mesures se singularisent également par des écartstypes très élevés, compris entre \pm 190 et \pm 310 (contre \pm 100 à \pm 120 pour les autres mesures, hors Poznan : tabl. 1). S'il reste là encore difficile de statuer sur la signification de ces incohérences en l'absence d'une analyse archéostratigraphique de l'ensemble de la séquence, il convient de signaler (1) que l'amplitude totale de l'intervalle c11-c17 variait par endroits de 16 à $22 \mathrm{~cm}$ (en CD2-3 : Fourment et Giraud 2012, p. 456), (2) que le vestige daté en couche 16 se situait dans un "véritable foyer en cuvette » dégagé en CD2-3 (Ibid.) et (3) que les industries lithiques des couches 11 , 16 et 27 renferment chacune 1 à 2 pièces à retouche solutréenne dont les états de surface ne témoignent pas de reprises ultérieures (Ducasse 2010, p. 34 et 197).

L'amorce récente d'un tel travail de réévaluation, mené par deux d'entre nous (CR et SD) autour des couches 31 à 29 , 28 et 27 , permet d'ores et déjà de montrer que s'ils existent (fig. 2 : 2 liens démontrés entre la c27 et la c29), les remontages inter-couches reliant l'ensemble solutréen aux premiers niveaux badegouliens restent extrêmement rares et ne traduisent probablement que l'existence de perturbations localisées et de faible ampleur, voire sont simplement liés aux difficultés de lecture stratigraphique (Clottes et Giraud 2012, p. 27). Dans la même perspective, le statut de la couche 28 , située à l'interface entre Solutréen supérieur et Badegoulien ancien, devait être rediscuté. Présente sur la quasi-totalité de la zone fouillée, décrite comme « (...) presque stérile, comme si l'abri avait été délaissé pendant quelque temps après le passage des Solutréens » (Clottes et Giraud 1989, p. 87), cette couche renferme des vestiges dont la projection stratigraphique montre la double appartenance : parfois nettement distincts des premières pièces cotées solutréennes mais en contact direct avec la nappe de vestiges de la couche 27 (i.e. Badegoulien ancien), parfois intercalés entre les couches 27 et 29 (i.e. Solutréen supérieur, cf. infra fig. 7B). Les tentatives de raccords et remontages entre ces trois couches sont venues confirmer le caractère " mixte » de la c.28, puisqu'au-delà de la présence conjointe de quelques fragments de pièces à retouches plates et couvrantes et de plusieurs éclats de bois de renne identiques aux déchets techniques badegouliens présentés en figure $3\left(n^{\circ} 2\right.$ à 6 ; Pétillon et Averbouh 2012), des liens physiques ont pu être clairement établis (fig. 2), entre les couches 27 et 28 d'une part (4 cas), et entre les couches 28 et 29 de l'autre ( 1 cas). Dans chaque cas plusieurs appariements (rapprochements litho-technologiques) ont également été documentés, renforçant l'hypothèse d'un assemblage « factice ", fruit de l'association d'éléments chrono-culturellement distincts.

L'intensification de la recherche systématique de raccords et remontages inter-couches et son extension aux niveaux concernés les plus récents (cas des couches 11 et 15/16) constituera l'un des principaux objectifs des analyses à venir.

\section{Une nouvelle série de dates par SMA}

À un cadre radiométrique cohérent et en très bonne résonnance avec les données jusqu'alors disponibles $\left({ }^{14} \mathrm{C}\right.$ classique : Bosselin et Djindjian 1988), succède donc aujourd'hui un cadre vieilli, resserré (entre 24,5 et 22 ka cal. BP une fois retranchées les dates de Poznan) et bien moins cohérent, sans équivalent publié sur le plan régional comme interrégional.

En 2011, dans le prolongement d'un travail mené autour de la diversité et de l'évolution des modalités de production de l'équipement badegoulien en bois de renne (Pétillon et Averbouh 2012 ; Pétillon et Sacchi à paraître), deux d'entre nous (JMP et SD) ont tenté la réalisation de plusieurs datations directes sur déchets de débitage, cela dans l'objectif de rediscuter de certaines associations technologiques (débitage par percussion versus double rainurage : Pétillon et Ducasse 2012). Parallèlement à la réévaluation de contextes ambigus (p.e. Lassac: Sacchi 2003 ; Reverdit : Bourdier et al. 2014 ; Les Harpons : Ducasse et Renard dir. 2013, p. 57-63), la constitution d'un référentiel de dates par SMA sur déchets techniques caractéristiques issus de contextes archéostratigraphiques fiables a pu être entamée. Dans ce cadre, les industries

(11) La date obtenue pour la couche 25, bien que très imprécise, ne peut être considérée comme aberrante une fois envisagée sa marge statistique à 2 sigmas (cf. tabl. 1 et fig. 1). Son intérêt reste donc limité. 


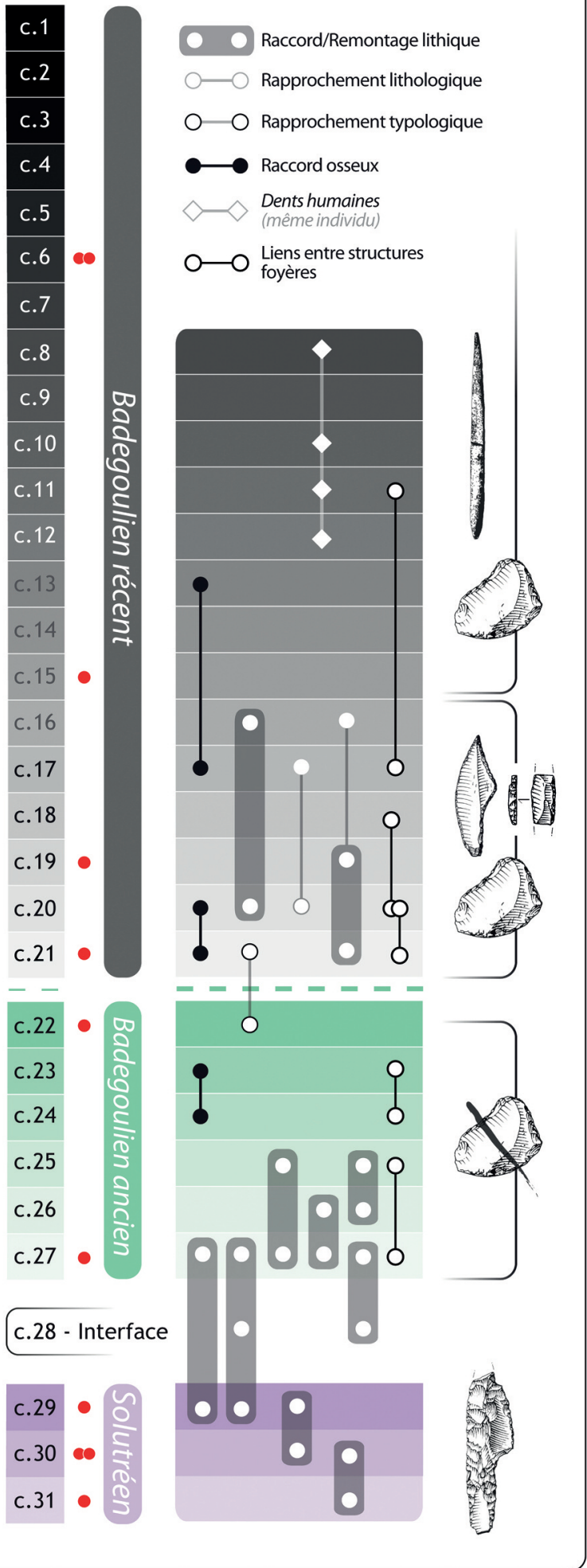

Figure 2 - Position stratigraphique des différentes mesures réalisées entre 2011 et 2013 (1 cercle rouge = 1 date) et rappel des liens typologiques et stratigraphiques mis en évidence au fil de la séquence (d'après Ducasse et Renard 2012, modifié).

Figure 2 - Stratigraphic position of the AMS datings made in 2011-2013 (1 red circle = 1 date) and summary of the typological and stratigraphical links through the sequence (after Ducasse and Renard 2012, modified). badegouliennes du Cuzoul-de-Vers ont été mises à contribution afin d'obtenir de nouveaux jalons chronologiques pour le débitage par percussion dont elles représentent l'un des exemples les mieux documentés à ce jour (Pétillon et Averbouh 2012). Initialement limitée à deux mesures ${ }^{14} \mathrm{C}$ confiées au laboratoire d'Oxford avec la volonté d'en publier les résultats rapidement (Pétillon et Ducasse 2012 : tableau 5, p 158), cette campagne de datation a finalement pu être étendue à sept mesures grâce à l'octroi de cinq dates supplémentaires via le programme ARTEMIS 2011. Enfin, dans le cadre du PCR SaM et de l'exercice 2012 d'ARTEMIS, quatre vestiges issus des couches 29 à 31 ont été soumis à datation dans le but de rediscuter de la diachronie des occupations solutréennes mais également d'obtenir des données de comparaison de « qualité » (cf. stratégie d'échantillonnage) égale à celles du Badegoulien (Ducasse et Renard 2014). Cette extension a donc été l'occasion de faire converger une question thématique liée à l'équipement osseux badegoulien et la réévaluation devenue nécessaire du cadre radiométrique de cette séquence de référence pour le DMG ouest-européen.

\section{Stratégies d'échantillonnage}

Pour le Badegoulien, six pièces techniques ont ainsi été sélectionnées en juin 2011 pour un total de sept prélèvements confiés aux laboratoires d'Oxford (fig. $3, n^{\circ} 1$ et 2 ) et de Lyon (datation à Saclay; $n^{\circ} 1$ et $n^{\circ} 3$ à 6 ). Quatre de ces pièces correspondent à des éclats de percussion liés au "façonnage » de la perche ( $n^{\circ} 2$ à 5), les deux pièces restantes regroupant une base de bois de chute sectionnée transversalement et portant un négatif d'éclat $\left(n^{\circ} 1\right)$ ainsi qu'une « bande » de tissu compact interprété comme un support potentiel ( $n^{\circ} 6$; Pétillon et Averbouh 2012, p. 380). Les questions alors posées dans le cadre de l'analyse des productions en bois de renne étant essentiellement centrées autour de la transition badegoulomagdalénienne (Pétillon et Ducasse 2012), les deux échantillons destinés au laboratoire d'Oxford ont été sélectionnés parmi les assemblages attribués au Badegoulien récent (tabl. 2). Outre l'exigence de ne choisir que des vestiges cotés, les seules véritables contraintes ont concerné leur état de surface mais aussi leurs dimensions et robustesse afin que les pièces soient les plus adaptées aux modalités de prélèvement décrites ciaprès. Ce choix s'est opéré au sein des couches 6 et 15 constituant deux des niveaux les plus denses en bois de renne (Pétillon et Averbouh 2012, p. 365). Ensuite, bien qu'elle ait eu lieu au même moment et ait concerné le même type de pièces, la sélection des vestiges à dater dans le cadre du programme ARTEMIS a suivi une logique propre et complémentaire. La partie supérieure de la séquence étant prise en charge par Oxford, il s'agissait d'échantillonner (1) la base de la séquence du Badegoulien récent marquée par la présence de pointes à cran à retouches abruptes originales (couches 16 à 21) et (2) le Badegoulien ancien (couches 22 à 27), rare à l'échelle du sud-ouest français et jusqu'ici très mal caractérisé sur le plan radiométrique (cf. supra). C'est donc en tenant compte de la représentativité de chaque ensemble, des liens 


\begin{tabular}{|c|c|c|c|c|c|c|c|c|}
\hline Réf. échantillon & Couche & Attribution & Matériau & Type d'échantillon & Code laboratoire & Age BP & Age cal. BP (2б) & Réf. fig. \\
\hline CZ 84 K4 c.6c' n²89 & 6 & Badegoulien récent & BdR & Base avec négatifs d'éclats & Lyon-9074 (SacA-28341) & $18620 \pm 100$ & $22757-22290$ & fig $2 n^{\circ} 1$ \\
\hline CZ 84 K4 c.6c' n²89 & 6 & Badegoulien récent & $\mathrm{BdR}$ & ase avec négatifs d'éclats & OxA 24963 & $18660 \pm 100$ & 22803-22335 & fig $2 n^{\circ} 1$ \\
\hline CZ 85 D 5 c. 15 a n²230 & 15 & Badegoulien récent & $\mathrm{BdR}$ & Éclat de percussion & OxA 24964 & $19180 \pm 110$ & $23468-22804$ & fig $2 n^{\circ} 2$ \\
\hline CZ 83 D4 c.19 nº1772 & 19 & Badegoulien récent & $\mathrm{BdR}$ & Éclat de percussion & Lyon-9076 (SacA-28343) & $18590 \pm 110$ & $22757-22216$ & fig $2 n^{\circ} 3$ \\
\hline CZ 85 J4 c.21c n 1555 & 21 & Badegoulien récent & $\mathrm{BdR}$ & Éclat de percussion & Lyon-9075 (SacA-28342) & $18860 \pm 110$ & $23003-22460$ & fig $2 n^{\circ} 5$ \\
\hline CZ 86 I5 c.22a nº36 & 22 & Badegoulien ancien & $\mathrm{BdR}$ & Bande corticale & Lyon-9077 (SacA-28344) & $18920 \pm 110$ & 23072-22489 & fig $2 n^{\circ} 6$ \\
\hline CZ 86 D5 c.27 nº1192 & 27 & Badegoulien ancien & $\mathrm{BdR}$ & Éclat de percussion & Lyon-9078 (SacA-28345) & $19150 \pm 110$ & $23446-22757$ & fig $2 n^{\circ} 4$ \\
\hline CZ 86 E5 c.29a nº1649 & 29 & Solutréen supérieur & Os & Métatarsien de renne & Lyon-10351 (SacA-33960) & $19410 \pm 100$ & 23668-23038 & fig $3 n^{\circ} 1$ \\
\hline CZ 86 I4 c.30 nº2472 & 30 & Solutréen supérieur & Os & Diaphyse OTM & Lyon 10352 (SacA 33961) & $19380 \pm 100$ & $23631-23016$ & fig $3 n^{\circ} 3$ \\
\hline CZ 85 B4 c.30 nº1291 & 30 & Solutréen supérieur & Os brûlé & Tibia de renne & Lyon 10353 (SacA 33962) & $19050 \pm 100$ & $23302-22608$ & fig $3 n^{\circ} 2$ \\
\hline CZ 86 J4 c.31 n²049 & 31 & Solutréen supérieur & Os & Diaphyse OTM & Lyon 10354 (SacA 33963) & $19320 \pm 100$ & $23566-22966$ & fig $3 n^{\circ} 4$ \\
\hline
\end{tabular}

Tableau 2 - Résultats des datations SMA réalisées entre 2011 et 2013 (BdR = Bois de renne). L'ensemble des calibrations a été réalisé avec l'aide du logiciel OxCal (v4.2.3 : Bronk Ramsey 2013) utilisant la courbe de calibration IntCal13 (Reimer et al. 2013).

Table 2 - AMS datings made in 2011-2013 (BdR = Antler). Calibration has been done with the OxCal program (v4.2.3: Bronk Ramsey 2013) using the IntCal13 calibration curve (Reimer et al. 2013).

typologiques et/ou physiques établis entre eux (fig. 2 ; Ducasse et Renard 2012 : figure 1, p.464) ${ }^{12}$, ainsi que de la présence en leur sein de déchets caractéristiques datables et cotés, que les couches 19, 21, 23 et 27 ont été préalablement choisies. Enfin, pour tester - et renforcer (cf. supra) - la fiabilité d'un cadre radiométrique alimenté par plusieurs laboratoires distincts, la base de bois de renne issue de la couche 6 a fait l'objet d'un second prélèvement pris en charge par les laboratoires de Lyon/Saclay.

L'informatisation des bordereaux de fouille n'ayant pas été entamée au moment de ce travail, la sélection n'a pu être appuyée par une projection systématique de chaque vestige. Elle a néanmoins été dans quelques cas menée en recherchant leur proximité planimétrique et altimétrique avec certains objets lithiques particuliers, comme c'est notamment le cas de l'éclat de percussion issu de la couche 21 , situé à proximité directe d'un fragment de pointe à cran badegoulienne (fig. $3, n^{\circ} 5 ; \mathrm{J} 4$ c.21c $n^{\circ} 1504$ : la " tranche » stratigraphique choisie en fig. $6 \mathrm{~A}$ ne permet cependant pas d'illustrer cette relation spatiale). Quoi qu'il en soit, la projection de l'ensemble des pièces concernées a été rendue possible a posteriori par le " cahier des charges " suivi lors de la sélection (fig. 6 et 7). Si elle ne manquera pas d'alimenter le travail archéostratigraphique d'ores et déjà planifié (cf. supra), l'étape de retour sur les carnets nous a au demeurant permis de corriger l'origine stratigraphique de l'une des pièces datées : la bande corticale sélectionnée en couche 23 d'après son marquage (fig. 3, $n^{\circ} 4$ ) s'est avérée, au regard des informations issues des bordereaux, appartenir à la couche 22 . Bien que cette réattribution n'ait, théoriquement, qu'un faible impact sur la stratégie de datation mise en œuvre (il s'agit dans les deux cas d'un ensemble attribué au Badegoulien ancien), notons que la couche 22 avait été volontairement écartée du processus de sélection compte-tenu de sa position intermédiaire entre Badegoulien ancien et récent mais aussi de la présence de quelques raclettes qui, sur le plan spatial, sont nettement associées aux « nappes » de raclettes documentées en couches 20 et 21 (fig. 2 ; Ducasse 2010, p. 230). Nous verrons que ces éléments de discussion éclairent efficacement l'interprétation que l'on peut actuellement faire du résultat ${ }^{14} \mathrm{C}$ obtenu à partir de cet échantillon (cf. infra).

In fine, précisons que la spécificité de cette sélection tient au fait qu'elle ne permet théoriquement pas de répondre à des questions d'ordre taphonomique dont, notamment, le problème posé par les mesures d'âge solutréen obtenues en couches 11 et 16 par exemple. En effet, le choix de ne dater que des vestiges techniques liés au débitage du bois de renne par percussion revient, au Cuzoulde-Vers, à dater les occupations badegouliennes dans la mesure où la méthode de production qui génère ce type de déchet n'a pas été documentée dans les niveaux solutréens (par ailleurs déficitaires en bois de cervidé travaillés : Pétillon et Averbouh 2012) ${ }^{13}$.

(12) Dans la mesure où le travail de critique taphonomique de la séquence badegoulienne n'est pas achevé, les données présentées en figure 2 doivent être considérées comme le reflet d'un état de la recherche susceptible d'évoluer. C'est ce qui explique ici l'absence de quantification exacte des raccords et remontages d'ores et déjà effectués, quantification dont la signification s'avère aujourd'hui limitée.

(13) Signalons néanmoins que des éclats de bois de renne existent bel et bien en contexte solutréen mais pourraient s'insérer dans des chaînes opératoires distinctes liées à des objectifs en partie différents (Baumann et Maury 2013 ; Chauvière et al. 2013 ). La datation directe de certains de ces éléments au Grand-Abri de Cabrerets, site quercinois fouillé au cours des années 1950 par A. Lemozi (1961), est récemment venue alimenter ce débat (Ducasse et Renard dir. 2013, p. 36). 


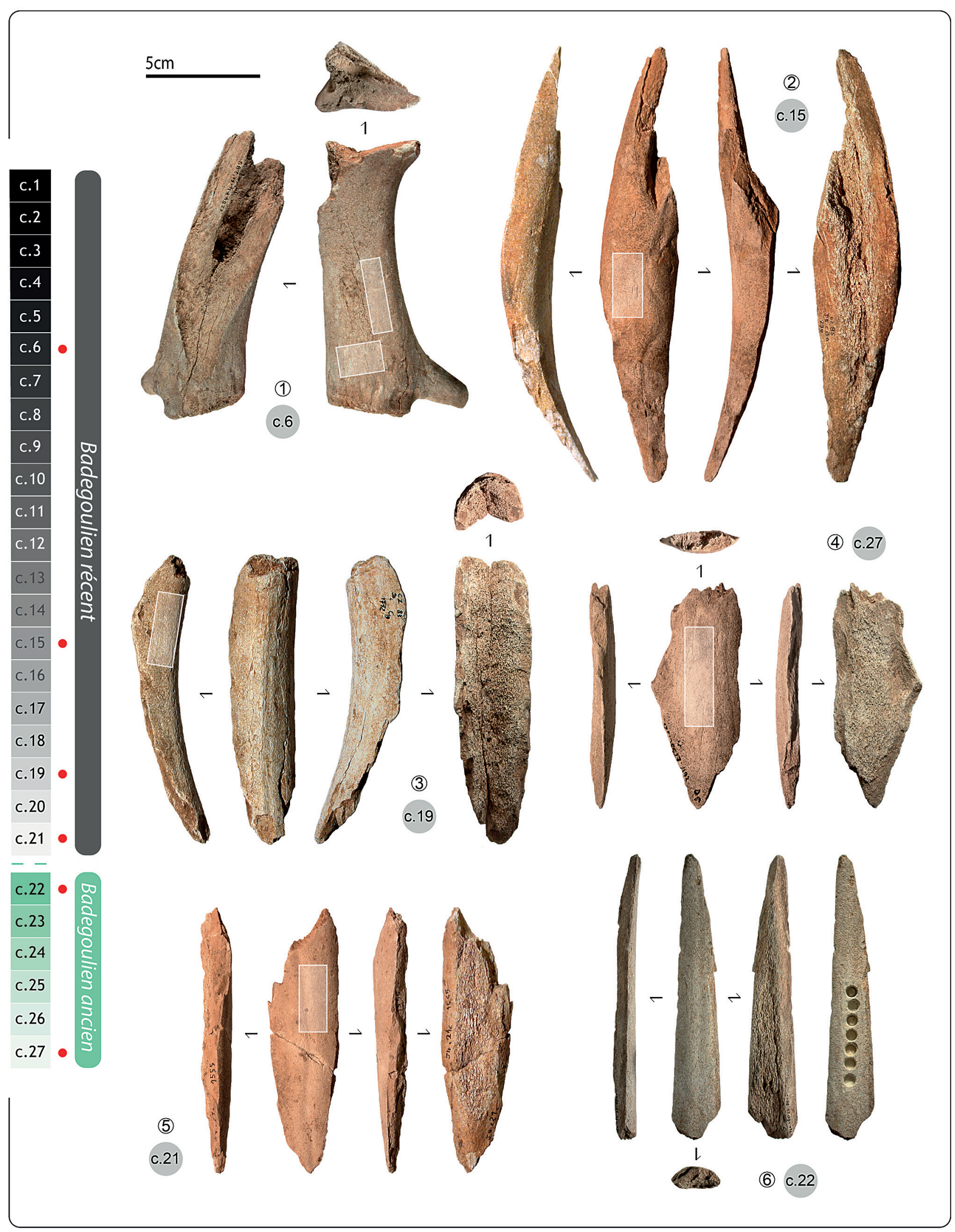

Figure 3 - Nature et position stratigraphique des pièces sélectionnées au sein de la séquence badegoulienne pour datation SMA $\left(n^{\circ} 1\right.$ : base de bois de chute présentant les stigmates d'un débitage par percussion lancée diffuse, $n^{\circ} 2$ à 5 : éclats de percussion, $n^{\circ} 6$ : «bande " corticale interprétée comme un support potentiel). Les surfaces délimitées par les rectangles blancs indiquent les zones prélevées ; remarquer la série de micro-forages visible sur la pièce $n^{\circ} 6$.

Figure 3 - Nature and stratigraphic position of the samples chosen for AMS dating within the badegoulian sequence ( $n^{\circ}$ 1: base of shed antler showing knapping stigmata; $n^{\circ} 2$ to 5 : antler flakes, $n^{\circ}$ 6: blank splinter). The white rectangles show the sampled areas; note the line of drillings on the sample $n^{\circ} 6$. 
Ainsi, devant l'extrême rareté de l'industrie osseuse solutréenne, par ailleurs peu caractéristique (Le Guillou 2012 ; Pétillon et Averbouh 2012), notre choix s'est dans ce cas portée sur des vestiges fauniques issus de chacune des trois couches. Effective en début d'année 2013, cette sélection a pu bénéficier, au contraire des niveaux badegouliens, d'une projection stratigraphique systématique de chaque vestige préalablement sélectionné, ceci dans le but premier de tester l'hypothèse selon laquelle les trois couches individualisées lors des travaux de terrain pourraient en réalité composer un ensemble unique et cohérent (Renard 2012) où se distingueraient des « zones d'activité » spécifiques à l'est et à l'ouest du gisement. Fondée sur des bases typologiques et technoéconomiques, sur l'interprétation des différents raccords et remontages effectués (cf. fig. 2) mais également sur des distributions spatiales " complémentaires » (Fourment et Giraud 2012, p.409), cette hypothèse est nuancée par des données ${ }^{14} \mathrm{C}$ contradictoires (cf. supra). C'est donc avec la collaboration de Jean-Christophe Castel que quatre vestiges osseux coordonnés (fig. 4A) ont été sélectionnés pour datation, et ce en fonction de leur position au regard de la répartition spatiale de chacune des couches (fig. 4B). Tandis qu'un métatarsien de renne (fig. $4 \mathrm{~A}, \mathrm{n}^{\circ} 1$ ) a été choisi en couche 29 - couche qui se développe essentiellement à l'est de l'abri -, deux vestiges ( $n^{\circ} 2$ et 3 ) l'ont été en couche 30 qui, quant à elle, correspond à deux concentrations disjointes corrélées à deux foyers, l'un situé à l'ouest de l'abri, l'autre à l'est. De fait, les deux pièces sélectionnées proviennent respectivement de l'une et l'autre de ces concentrations (fig. 4B ; un tibia de renne en couche 30-est ainsi qu'un fragment de diaphyse d'ongulé de taille moyenne en couche 30-ouest). Enfin, le quatrième et dernier objet concerné ( $n^{\circ} 4$ : diaphyse d'ongulé de taille moyenne) provient de la base de la couche 31 , couche localisé à l'est de l'abri et interprétée comme le prolongement du foyer présent dans cette zone en couche 30 (cf. note 6).

\section{Modalités de prélèvement}

Si la nature des pièces sélectionnées dans les trois couches solutréennes ne présentait pas de contraintes majeures en termes de conservation (i.e. vestiges fauniques peu " sensibles », prélèvements directement réalisés par le laboratoire de Lyon), il en est allé tout autrement des éléments badegouliens pour lesquels il convenait de limiter au maximum les destructions inhérentes aux prélèvements. De fait, et bien que le statut patrimonial de ces pièces (i.e. déchets de débitage en bois de renne) se distingue de celui d'éventuels objets finis parfois sélectionnés pour datation (par ex. Aujoulat et al. 1998 ; Barandiaran 1988 ; Chauvière et al. 2006 ; Pétillon et al. sous presse ; Szmidt et al. 2009 ; Tisnerat-Laborde, Valladas, Ladier 1997), les modalités de prélèvement mises en œuvre ont dans la mesure du possible consisté à préserver l'intégrité morphologique, dimensionnelle et technologique de chaque vestige (fig. 3). Cette condition sine qua non est respectée afin de permettre un réexamen technologique de ces pièces et, ainsi, nourrir une éventuelle critique des résultats et interprétations présentés ici (voire d'effectuer des contre-expertises radiométriques à partir des mêmes pièces).

Un protocole spécifique de prélèvement avant soumission au laboratoire a donc été mis en place, directement inspiré du procédé développé par Laurent Brou (Brou 2006 ; Ducasse et Renard dir. 2013, p. 32-34 avec quelques adaptations). Après prise d'informations morphométriques et technologiques puis couverture photographique, chaque pièce a été soumise à la même suite d'opérations : (1) sélection de la zone la mieux adaptée (i.e. bonne épaisseur de tissu compact, zone non fissurée pour éviter une fragmentation de l'objet, absence de stigmates techniques lisibles) ; (2) nettoyage de la zone choisie par abrasion de la couche externe de l'objet, potentiellement contaminée (outil utilisé : foret à bois de $3 \mathrm{~mm}$ de diamètre monté sur une mini-perceuse à main) ; (3) prélèvement de la matière à dater par micro-forages successifs (p.e. fig. $3, n^{\circ} 6$ ) afin d'obtenir plusieurs échantillons solides dont la masse cumulée atteint entre 450 et $1040 \mathrm{mg}$ selon les pièces (outils utilisés : trépan de $7 \mathrm{~mm}$ de diamètre, balance de précision). Ces trois étapes ont été répétées pour chacune des pièces, les forets et trépans ayant été nettoyés par immersion dans un bac à ultrason entre chaque série de prélèvements afin d'éviter d'éventuelles pollutions inter échantillons. Les sept échantillons ainsi obtenus ont été stockés dans des tubes Eppendorf stériles et confiés sous cette forme aux laboratoires dateurs.

\section{Résultats et discussion}

L'ensemble des prélèvements soumis a donné lieu à datation. Les 11 résultats (tabl. 2 et fig. $5 \mathrm{~A}$ ) montrent un même niveau de précision, les écarts-types étant compris entre \pm 100 et \pm 110 , et ce quel que soit le laboratoire concerné. Une fois calibrées, les mesures forment un ensemble relativement cohérent en comparaison des données acquises en 2001 (fig. 1B, à droite), à l'exception des échantillons issus des couches 15 - qui livre un âge aberrant dont la cause ne peut être à ce stade clairement identifiée (fig. 6D) ${ }^{14}$ - et 30 -est, cas sur lequel nous reviendrons. Pour le Badegoulien, cette bonne cohérence résulte en partie de la mise en œuvre d'une stratégie d'échantillonnage étrangère aux questions d'ordre taphonomique (pour lesquelles cette démarche reste, dans le cas présenté ici, peu efficiente, cf. supra) mais adaptée aux objectifs et questions posés préalablement. Elle transcende par ailleurs la double origine des mesures (tabl. 2 : Oxford $\mathrm{N}=2$; Lyon/Saclay $\mathrm{N}=5$ ) puisque les datations réalisées par chacun des deux laboratoires à partir d'échantillons issus du même vestige (cf. supra :

(14) Remarquons qu'elle renvoie à la mesure incohérente obtenue en couche 16 lors de la première série par SMA (fig. 1B), qu'elle présente un niveau de précision égal à celui des autres mesures réalisées entre 2011 et 2012. 


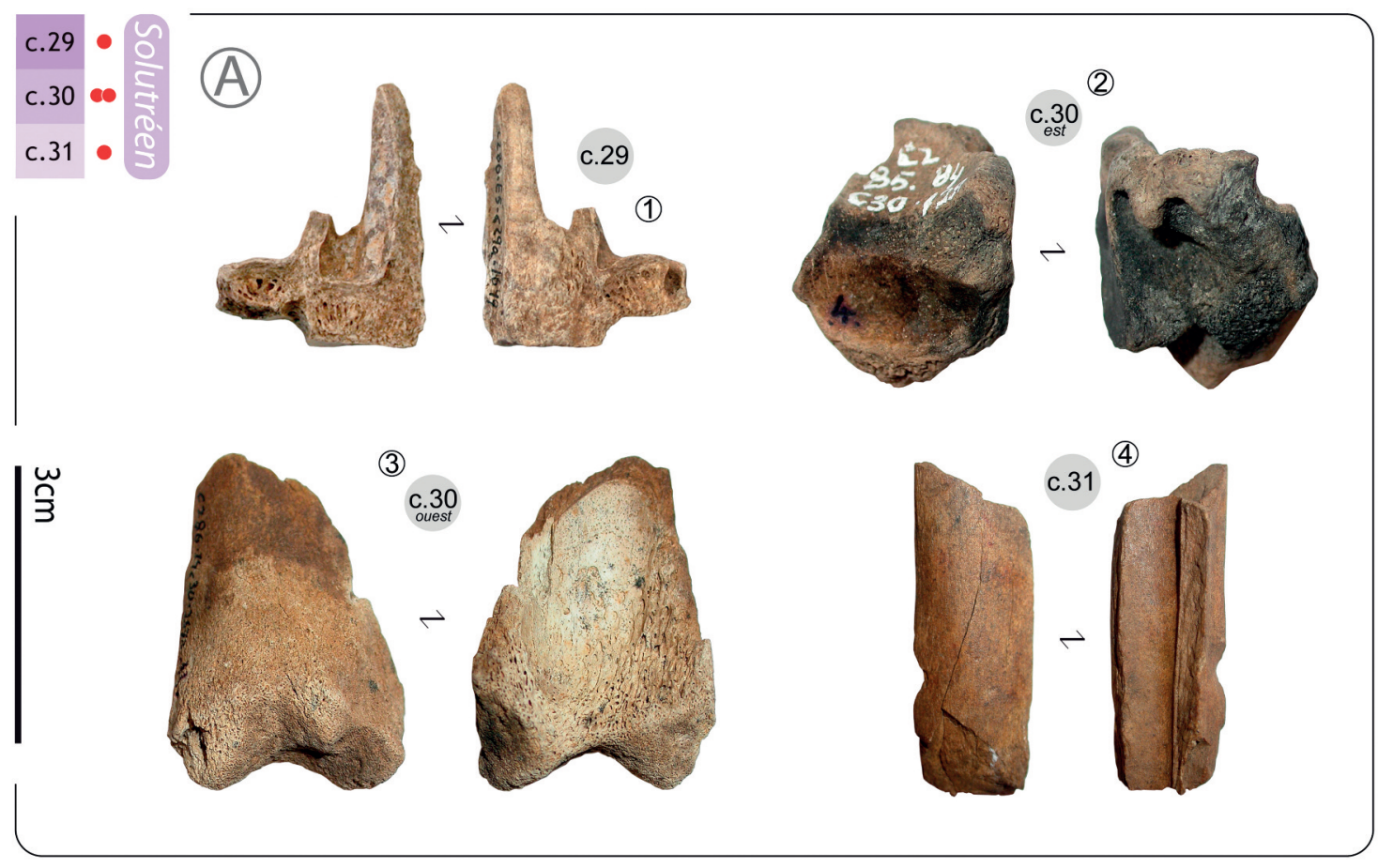

(B)

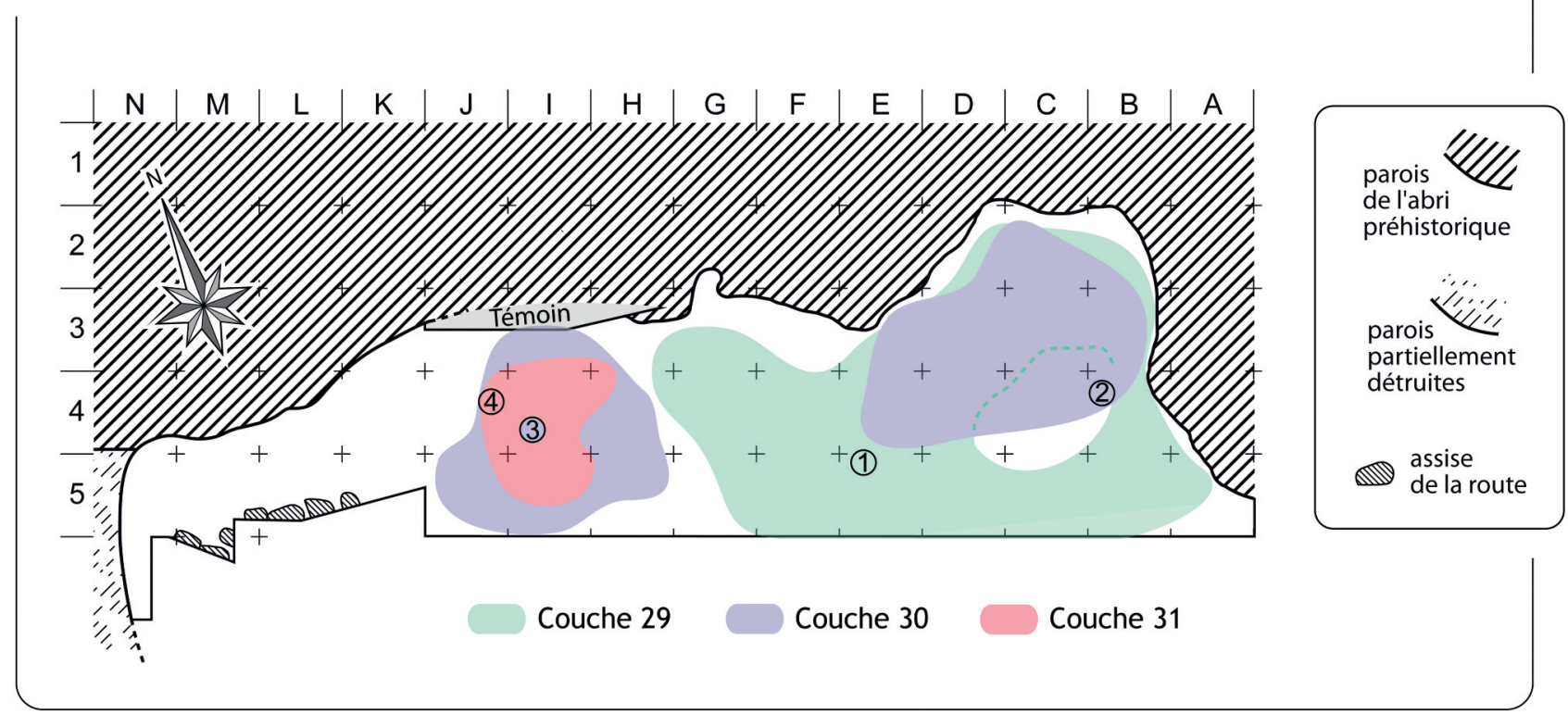

Figure 4 - Nature et localisation des pièces sélectionnées au sein de la séquence solutréenne pour datation SMA ( $n^{\circ} 1:$ métatarsien de renne ; $n^{\circ} 2$ : extrémité distale de tibia de renne brûlée ; $n^{\circ} 3$ : fragment distal de diaphyse d'un jeune ongulé de taille moyenne; $n^{\circ} 4$ : fragment de diaphyse d'ongulé de taille moyenne; $n^{\circ} 2$ à 4 : clichés J.-C. Castel).

Figure 4 - Nature and location of the samples chosen for AMS dating within the solutrean sequence ( $n^{\circ}$ 1: reindeer metatarsal; $n^{\circ}$ 2: burnt reindeer distal epiphysis tibia; $n^{\circ}$ 3: juvenile medium size ungulate distal shaft; $n^{\circ} 2$ and 4: photos J.-C. Castel).

couche 6) ont donné des résultats strictement équivalents (fig. $5 \mathrm{~A}: 18620 \pm 100 \mathrm{BP}$ et $18660 \pm 100 \mathrm{BP}$ ). Ces deux mesures, tout comme la nouvelle date obtenue pour la couche 29 (19410 $\pm 100 \mathrm{BP})$, sont à peu de choses près identiques aux résultats obtenus pour les mêmes ensembles en 2001 via le laboratoire d'Oxford (tabl. 1, $n^{\circ} 8$ : $\left.18730 \pm 110 \mathrm{BP}, \mathrm{n}^{\circ} 19: 19510 \pm 110 \mathrm{BP}\right)$. Ce premier élément offre la garantie d'un excellent niveau de comparabilité qui permet d'exclure le facteur "laboratoire » des discussions menées autour de cette nouvelle série de dates, et ce tout en autorisant leur intégration au corpus SMA déjà disponible. 

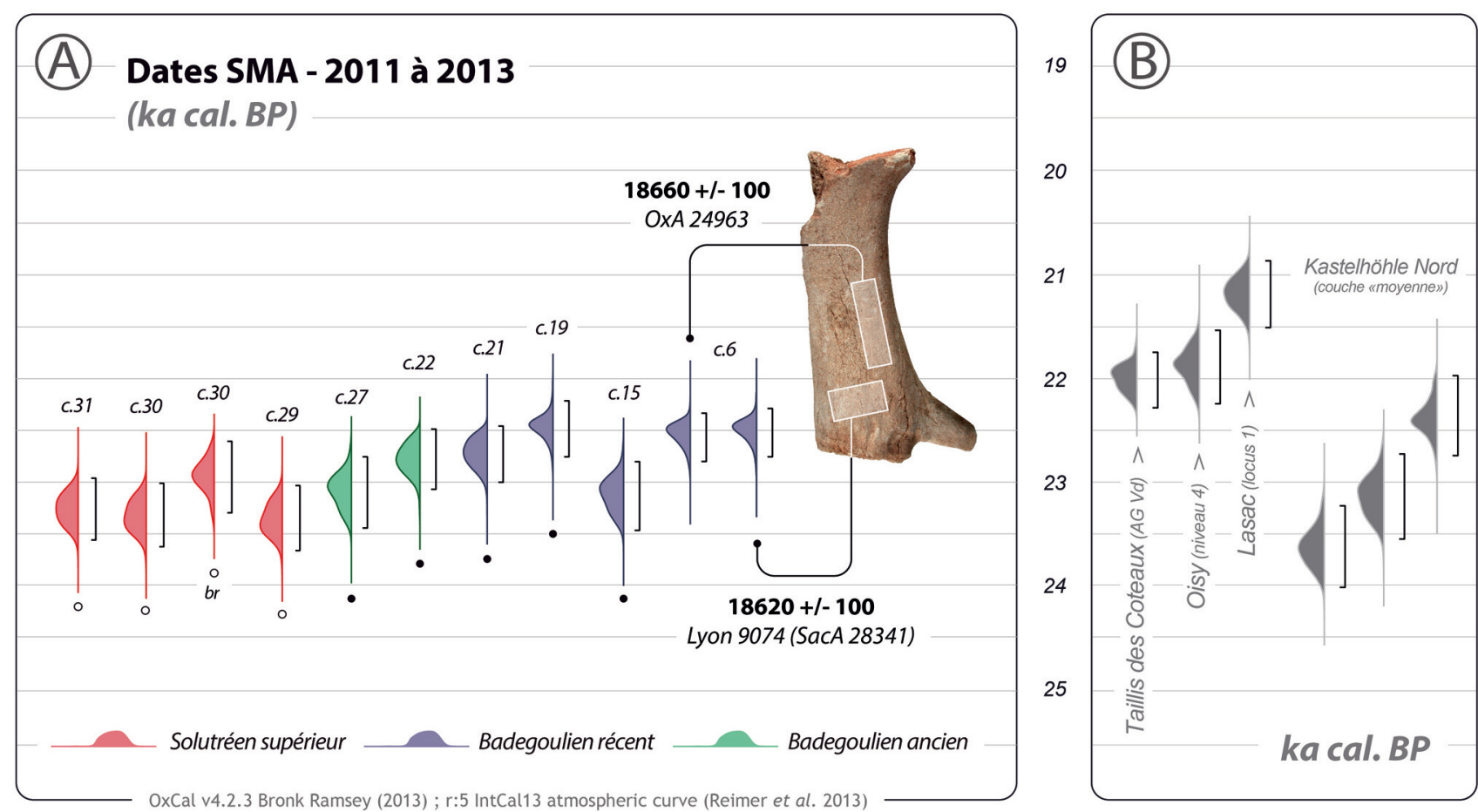

Figure 5 - Résultats de la campagne de datation SMA 2011-2013. A : Bilan des 11 nouvelles dates calibrées. Notez la similarité des mesures obtenues à Oxford et Lyon/Saclay à partir de deux échantillons prélevés sur le même vestige (couche 6). B : Sélection de dates ${ }^{14} \mathrm{C}$ (SMA) obtenues pour le Badegoulien à raclettes français (d'après Debout et al. 2012 ; Pétillon et Ducasse 2012 ; Primault et al. 2007) ainsi que pour les industries à affinités badegouliennes d'Europe centrale (d'après Terberger 2013).

Figure 5 - Results of the 2011-2013 AMS radiocarbon dates. A: Summary of the 11 calibrated new dates. Note the complete identity of the ${ }^{14} \mathrm{C}$ measurements made by Oxford and Lyon/Saclay from the same antler waste (layer 6). B: Comparison with a selection of ${ }^{14} \mathrm{C}$ dates (AMS) from raclette-yielding assemblages from the French Badegoulian (data from Debout et al. 2012; Pétillon and Ducasse 2012; Primault et al. 2007) and AMS radiocarbon dates from Badegoulian-like assemblages of Central Europe (data from Terberger 2013).

\section{Confirmation et affinement du cadre SMA : le plus vieux badegoulien ouest-européen}

L'apport majeur de ces nouveaux repères ${ }^{14} \mathrm{C}$ pour la séquence badegoulienne réside dans la confirmation de l'ancienneté des occupations ainsi que du resserrement chronologique introduit par les premières dates obtenues par SMA. De fait, entre la couche 6 qui constitue l'un des premiers véritables niveaux badegouliens (cf. note 7), et la couche 27 qui correspond au niveau le plus ancien attribuable à ce techno-complexe, l'amplitude statistique totale des occupations s'élève à environ 1300 ans cal. BP (tabl. 2 : entre ca. 23,5 et 22,2 ka cal. $\mathrm{BP}^{15}$ ) contre environ 3800 ans cal. BP en ${ }^{14} \mathrm{C}$ classique (tabl. 1 : couches 5 à 24). Un court moment donc, qui plus est situé dans une chronologie décidemment ancienne, bien plus que ce qu'indiquaient les dates ${ }^{14} \mathrm{C}$ classiques. Tandis que régionalement la date obtenue pour le niveau de Badegoulien à raclettes du Petit Cloup Barrat s'inscrit dans une fourchette chronologique similaire (Chauvière et al. 2008 ; Ducasse et al. 2011), les mesures par SMA disponibles pour le Badegoulien français ${ }^{16}$ donnent des âges généralement plus récents, globalement situés entre 22,5 et 21 ka cal. BP (fig. 5B ; p.e. Debout et al. 2012 ; Chehmana, Debout, Bodu 2013 ; Pétillon et Ducasse 2012 ; Primault et al. 2007 ; Sacchi, Brulé, Ducasse ed. à paraître). En attendant que le corpus de datation par SMA s'étoffe, le Badegoulien du Cuzoul-de-Vers se pose donc aujourd'hui comme le plus ancien d'Europe de l'ouest, situé dans une chronologie peu ou prou similaire à celle des industries d'affinité badegoulienne documentées en Europe centrale (fig. 5B ; Oberlin et Valladas 2012 ; Kozlowski et al. 2012 ; Sedlmeier 2010 ; Terberger 2013).

(15) Laps de temps estimés à partir des limites inférieure et supérieure des intervalles de confiances obtenus pour la date la plus ancienne (c.27) et la date la plus récente du Badegoulien (c. 6 ; tabl. 2).

(16) En très grande partie réalisées par le laboratoire de Lyon via le programme ARTEMIS. 

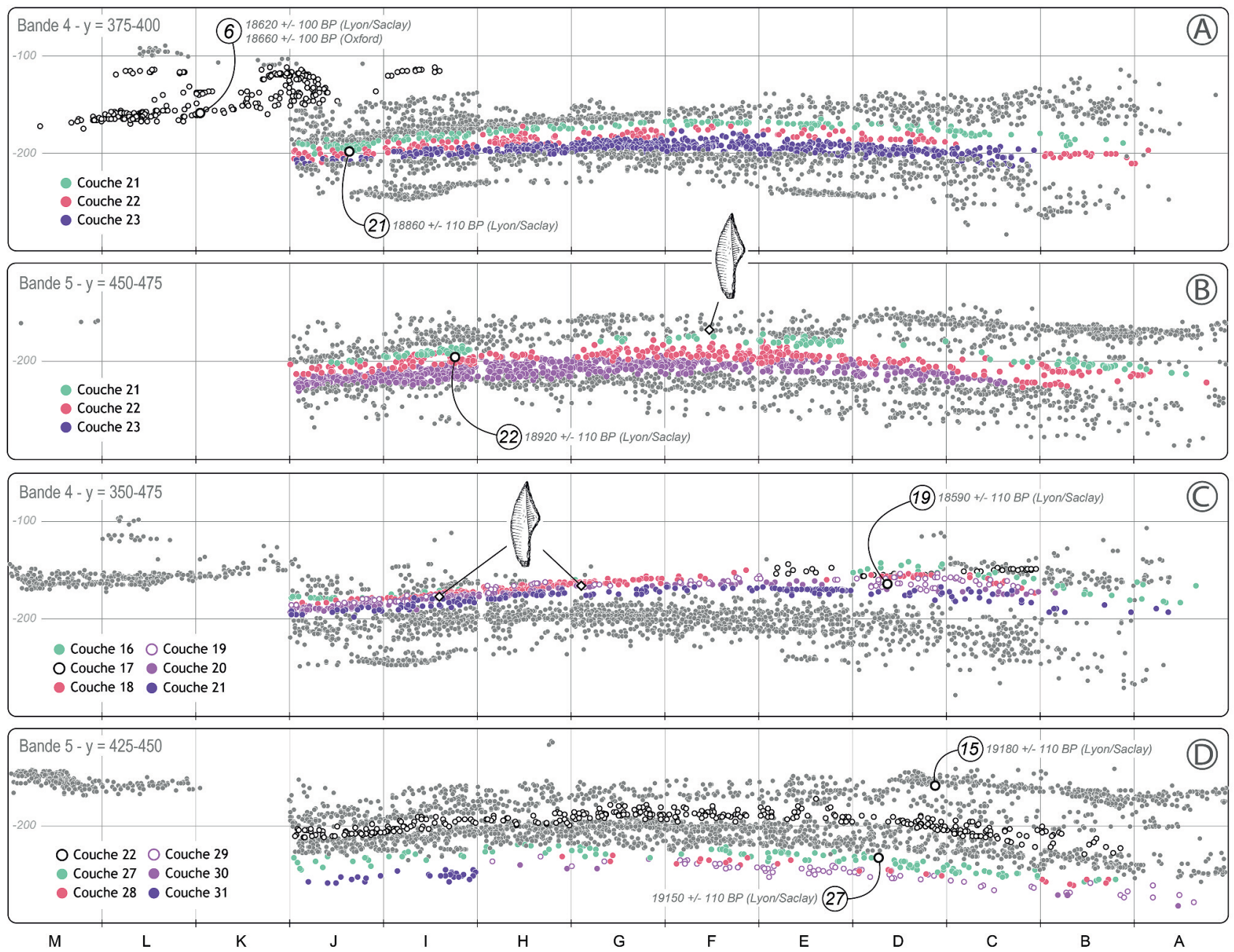

Figure 6 - Projection stratigraphique des éléments badegouliens datés (coupes frontales, tranches de $25 \mathrm{~cm}$ de largeur).

Figure 6 - Stratigraphic projection of the 6 dated pieces from the badegoulian levels (coronal planes, slice thickness $25 \mathrm{~cm}$ ).

\section{Une chronologie resserrée pour les niveaux solutréens}

Les quatre dates obtenues pour le Solutréen supérieur (tabl. 2) apportent des éléments de discussion importants - si ce n'est décisifs - à la compréhension de cet ensemble. Elles témoignent en effet d'une forte homogénéité et tendent à nourrir l'hypothèse, proposée à partir de l'analyse techno-économique et taphonomique des industries lithiques (cf. supra), d'un assemblage constitué dans une chronologie resserrée liée à un nombre très limité d'occupations (une seule et unique occupation ?). Trois d'entre elles, issues de l'ensemble des trois couches, se superposent parfaitement (fig. $5 \mathrm{~A}$ en rouge), se faisant par ailleurs l'écho de la date SMA déjà obtenue pour la couche 29 et considérée, par comparaisons inter-sites, comme la plus cohérente des deux dates disponibles. Remarquons également que, contrairement au Badegoulien où datations classiques et datations SMA sont généralement discordantes (cf. supra), ces nouvelles dates entrent en parfaite cohérence avec la mesure réalisée par ${ }^{14} \mathrm{C}$ classique en couche 30 (tabl. 1 : $19400 \pm 210$ BP), pourtant issue comme les autres d'un lot d'esquilles osseuses. Il est tentant, dans ce cas, d'y voir là un autre indice de la grande homogénéité chronologique de l'ensemble 29-30-31 qui semble s'insérer dans une fourchette globalement comprise entre 23 et $23,5 \mathrm{ka}$ cal. BP. Seule note discordante, la mesure obtenue pour la couche 30 a fourni un âge sensiblement plus jeune (19050 \pm 100 BP ; fig. 7C) qui, s'il trouve des correspondances à l'échelle régionale (Ducasse et Renard 2014) et interrégionale (Hinguant et Biard 2013 ; Bodu, Dumarçay, Naton 2014), s'accorde a priori mal avec la position stratigraphique de l'objet daté (fig. 7B ; base de la cuvette BC3-4 : Fourment et Giraud 2012, p. 411). Considérant (1) la nature du matériau daté, distincte des trois autres (tabl. 2 : os brûlé) et (2) l'insertion - certes partielle - de cette date au sein du groupe formé par les trois premières une fois la mesure calibrée (fig. 5A), 

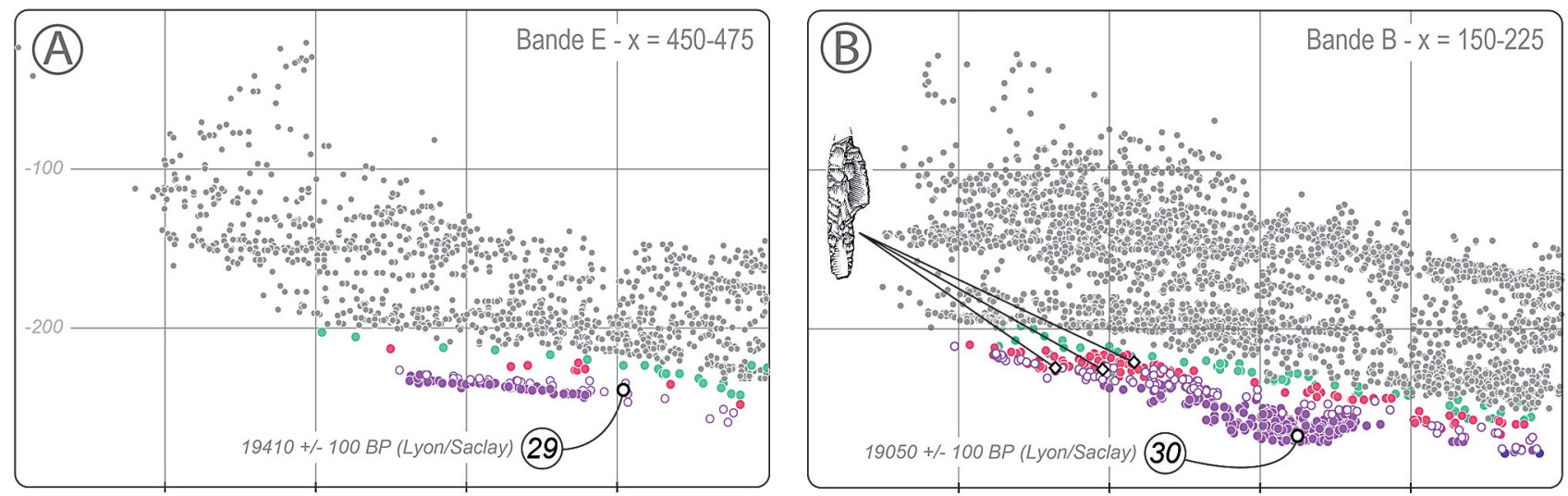

- Couche 31 Couche 30 O Couche 29 Couche 28 Couche 27
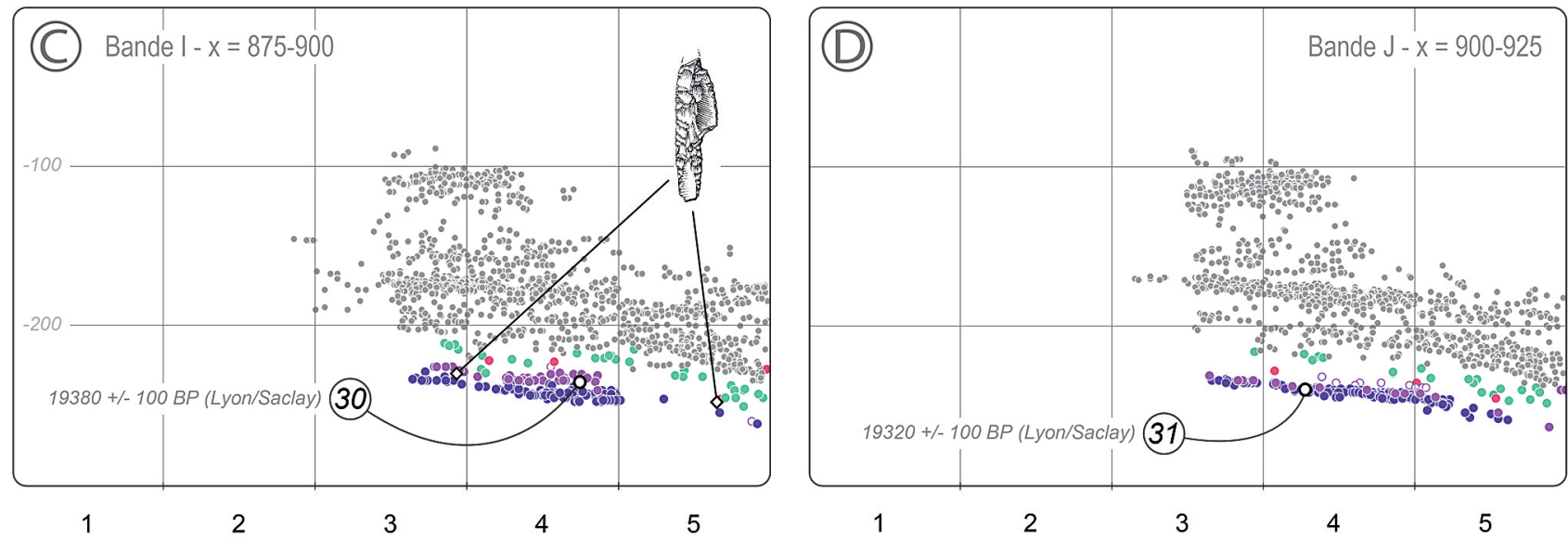

Figure 7 - Projection stratigraphique des éléments solutréens datés (coupes sagittales, tranches de 25 ou $75 \mathrm{~cm}$ de largeur).

Figure 7 - Stratigraphic projection of the 4 dated pieces from the solutrean levels (sagittal planes, slice thickness 25 or $75 \mathrm{~cm}$ ).

l'hypothèse d'une occupation solutréenne plus récente ou bien celle d'une contamination de ce niveau par des vestiges d'âge badegouliens doivent être considérées avec prudence en l'absence d'indices concrets.

Enfin, notons que la date obtenue pour la couche 31 $(19320 \pm 100 \mathrm{BP})$ et dont l'échantillon a été prélevé à la base du foyer en J4 (1) invalide l'attribution à l'ensemble solutréen de la date SMA anciennement fournie pour la couche 31 (20110 \pm 180 BP, GifA 101440) et (2) concorde parfaitement avec la date fournie par l'échantillon sélectionné pour la couche 30 sus-jacente en 14 (19380 \pm 100 BP). Si nous n'expliquons pas l'ancienneté de la date obtenue en 2001 par le laboratoire de Gif-sur-Yvette en c.31 (cf. supra), remarquons que cette nouvelle série de mesures tend à isoler un peu plus encore ce résultat peu cohérent avec les données archéologiques.

\section{Variations diachroniques des équipements et chronologie ${ }^{14} \mathrm{C}$ : quel degré de résolution?}

Nous avons vu qu'au sein de cette chronologie resserrée, la séquence du Cuzoul-de-Vers témoignait de changements plus ou moins profonds des équipements lithiques et osseux (Clottes, Giraud, Chalard dir. 2012 ; Ducasse et Renard 2012). Qu'ils soient typologiques ou techno-économiques, ces changements s'expriment tant à l'échelle intraculturelle (transition Badegoulien ancien/Badegoulien récent et variation diachronique des équipements au cours du Badegoulien récent) qu'interculturelle (transition Solutréen/Badegoulien). L'enjeu de ce renouvellement du cadre radiométrique réside également, nous l'avons vu, dans l'obtention de mesures ${ }^{14} \mathrm{C}$ susceptibles de mieux documenter la durée de ces différentes phases d'occupation mais aussi leurs bornes respectives.

Compte tenu de ces objectifs et forts de l'analyse critique de l'ensemble des dates SMA aujourd'hui disponibles pour le Cuzoul-de-Vers (cf. supra), nous avons pris le parti d'exclure des discussions qui suivront :

(1) les dates obtenues à partir de lots d'esquilles osseuses (c.6 et 11 : tabl. $1, n^{\circ} 9$ et 11 ) qui renvoient aux mêmes modalités d'échantillonnage que les datations classiques et, du même coup, présentent les mêmes biais (possibles dates « moyennées »); 
(2) les mesures qui, bien que fiables sur le plan physicochimique, ont donné des résultats incohérents difficilement interprétable à ce stade (c.11, 16 et $31:$ tabl. $1, n^{\circ} 10,13$ et 20 ; c.15 de la série 2011-2012 : tabl. 2) ;

(3) les mesures dont l'écart-type est supérieur à \pm 200 ( \pm 310 pour les couches 25 et $26, \pm 240$ pour la couche 27 : tabl. 1, $\mathrm{n}^{\circ} 16$ à 18) qui, bien trop imprécises (probabilités étalées sur environ 1500 ans cal BP à 2 sigma), ne nous sont d'aucune utilité au regard des questions posées ici.

Quinze mesures ont donc été retenues sur les 24 réalisées par SMA entre 2001 et 2013 pour l'ensemble des niveaux. Sept dates correspondent au Badegoulien récent (5 couches datées entre la c. 6 et la c.21), 3 dates au Badegoulien ancien (entre la c.22 et la c.27) et 5 dates au Solutréen supérieur (c.29 à 31), de sorte que, théoriquement, les bornes de chacune de ces subdivisions bénéficient d'un repère radiométrique. Une fois combinées les deux mesures obtenues à partir d'un même vestige issu de la couche 6 (fig. $8: 18640 \pm 71$ BP ; outil «R_Combine » du logiciel OxCal [version 4.2.3 : Bronk Ramsey 2013], utilisé avant calibration), la calibration des 14 dates retenues (tabl. 3) permettent de situer le Badegoulien récent dans un intervalle compris entre 23 et 22,3 ka cal. BP, le Badegoulien ancien se développant quant à lui entre 23,5 et 22,5 ka cal. BP. Comme l'illustre la figure 9 , ce très fort recouvrement entre Badegoulien ancien et récent est essentiellement dû à la mesure obtenue pour la couche $22 \mathrm{a}$, identique à celle aujourd'hui disponible pour la couche 21. Or, compte tenu (1) des éléments contextuels rappelés précédemment au sujet de la couche 22 et (2) de la projection stratigraphique de l'élément daté qui montre sa grande proximité spatiale avec

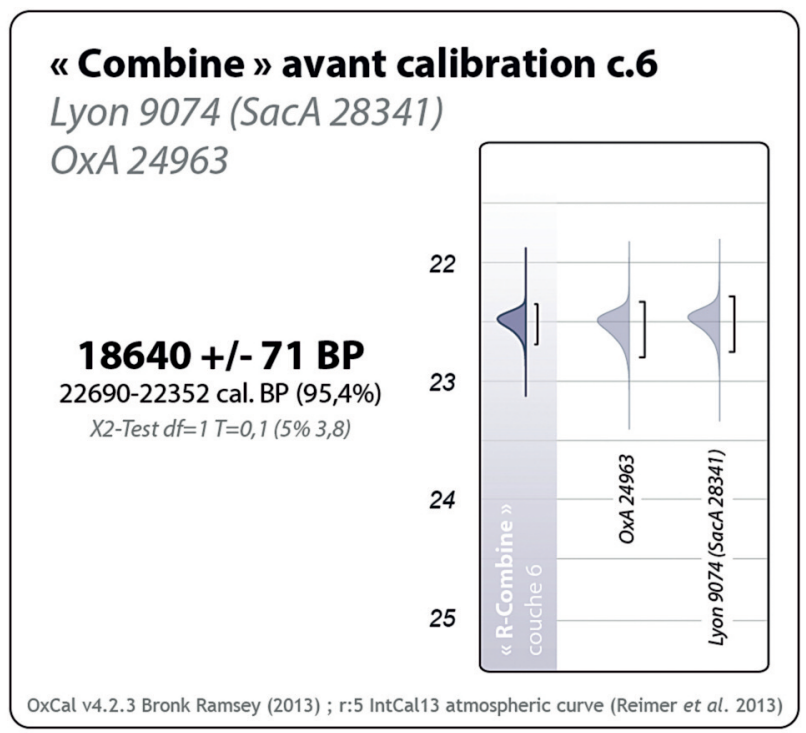

Figure 8 - «Combine » et calibration des deux dates SMA obtenues sur le même bois de renne issu de la couche 6 (OxCal v4.2. : Bronk Ramsey 2013).

Figure 8 - «Combine » and calibration of the two ${ }^{14} \mathrm{C}$ measurements from the same antler waste from layer $6(\mathrm{OxCal}$ v4.2. : Bronk Ramsey 2013).

le premier niveau à raclettes (c.21 : fig. 6B), I'hypothèse que cette mesure date en réalité la base du Badegoulien récent nous semble tout à fait soutenable. Ainsi, au Cuzoul-deVers, les deux seuls repères ${ }^{14} \mathrm{C}$ susceptibles de correspondre sans équivoque aux industries

\begin{tabular}{|c|c|c|c|c|c|c|}
\hline Couche & Code laboratoire & Attribution & Matériau & Type d'échantillon & Age BP & Age cal. BP $(2 \sigma)$ \\
\hline 6 & $\begin{array}{c}\text { Lyon-9074 (SacA-28341) } \\
\text { et OxA } 24963\end{array}$ & Badegoulien récent & $\mathrm{BdR}$ & Base avec négatifs d'éclats & $18640 \pm 71$ & $22690-22352$ \\
\hline 6 & OxA-10955 (Lyon-1674) & Badegoulien récent & Os & Diaphyse OTM & $18730 \pm 110$ & $22889-22383$ \\
\hline 15 & OxA-10974 (Lyon-1676) & Badegoulien récent & Os & Diaphyse OTM & $18730 \pm 100$ & 22871-22391 \\
\hline 18 & OxA-11118 (Lyon-1681) & Badegoulien récent & Os & Diaphyse OTM & $19020 \pm 110$ & $23262-22555$ \\
\hline 19 & Lyon-9076 (SacA-28343) & Badegoulien récent & $\mathrm{BdR}$ & Éclat de percussion & $18590 \pm 110$ & $22757-22216$ \\
\hline 21 & Lyon-9075 (SacA-28342) & Badegoulien récent & BdR & Éclat de percussion & $18860 \pm 110$ & $23003-22460$ \\
\hline $22 \mathrm{a}$ & Lyon-9077 (SacA-28344) & Badegoulien ancien & BdR & Bande corticale & $18920 \pm 110$ & $23072-22489$ \\
\hline $22 b$ & OxA-10976 (Lyon-1678) & Badegoulien ancien & Os & Diaphyse OTM & $19280 \pm 120$ & $23563-22901$ \\
\hline 27 & Lyon-9078 (SacA-28345) & Badegoulien ancien & $\mathrm{BdR}$ & Éclat de percussion & $19150 \pm 110$ & $23446-22757$ \\
\hline 29 & OxA-11220 (Lyon-1682) & Solutréen supérieur & Os & Diaphyse OTM & $19510 \pm 110$ & $23836-23124$ \\
\hline 29 & Lyon-10351 (SacA-33960) & Solutréen supérieur & Os & Métatarsien de renne & $19410 \pm 100$ & $23668-23038$ \\
\hline 30 & Lyon 10352 (SacA 33961) & Solutréen supérieur & Os & Diaphyse OTM & $19380 \pm 100$ & $23631-23016$ \\
\hline 30 & Lyon 10353 (SacA 33962) & Solutréen supérieur & Os brûlé & Tibia de renne & $19050 \pm 100$ & $23302-22608$ \\
\hline 31 & Lyon 10354 (SacA 33963) & Solutréen supérieur & Os & Diaphyse OTM & $19320 \pm 100$ & $23566-22966$ \\
\hline
\end{tabular}

Tableau 3 - Bilan des datations SMA retenues pour la discussion (BdR = Bois de renne). L'ensemble des calibrations a été réalisé avec la version 4.2.3 du logiciel OxCal (Bronk Ramsey 2013) utilisant la courbe de calibration IntCal13 (Reimer et al. 2013).

Table 3- AMS datings selected for discussion $(B d R=$ Antler). Calibration has been done with the OxCal program (v4.2.3: Bronk Ramsey 2013) using the IntCal13 calibration curve (Reimer et al. 2013). 
attribuées au Badegoulien ancien placent cet épisode entre 23, 5 et 23 ka cal. BP. Si, en considérant cette hypothèse, la marge de recouvrement existant entre les deux phases du Badegoulien se réduit donc considérablement, la transition solutréo-badegoulienne conserve de son côté toute sa nébulosité (Oberlin et Valladas 2012). Pourtant épargnée par d'éventuels plateaux ${ }^{14} \mathrm{C}$ depuis la publication de la courbe IntCal13 (Reimer et al. 2013), elle se caractérise par un chevauchement radiométrique incompressible qui englobe l'ensemble du Badegoulien ancien. Si hiatus d'occupation il y a eu entre derniers Solutréens et premiers Badegouliens en Aquitaine (p.e. Bosselin et Djindjian 1997 ; Djindjian 2000 ; hypothèse fondée en grande partie sur un corpus de dates non calibrées et obtenues par la méthode classique), admettons donc que le ${ }^{14} \mathrm{C}$ ne constitue à ce jour pas l'outil le plus efficace pour mettre en lumière un tel phénomène, si tant est qu'il ait existé. Au contraire, malgré l'imprécision des résultats, ces données tendent à montrer non seulement l'ancienneté mais également la relative rapidité de ce moment de reconfiguration des normes technoéconomiques. Reste qu'au Cuzoul-de-Vers le Badegoulien ancien ne semble pas perdurer bien au-delà de $23 \mathrm{ka}$ cal. $\mathrm{BP}$, limite à partir de laquelle se développent en France les industries à raclettes du Badegoulien récent (type d'ensemble connu jusqu'aux alentours de 21 ka cal. BP : Langlais et al. 2010 ; Ducasse 2012 ; Pétillon et Ducasse 2012).
Ce canevas chronologique confirme donc la nuance qu'il convient d'apporter à la signification de la dilatation stratigraphique de cette séquence, à la succession des nombreux niveaux sédimentaires qui la composent (cf. supra). Ainsi, le resserrement des mesures ${ }^{14} \mathrm{C}$ témoigne d'une phase d'occupation de l'abri bien plus courte qu'envisagée à l'origine, tandis que les différents liens physiques établis entre certaines des couches (fig. 2) permettent d'individualiser des ensembles cohérents et significatifs sur les plans typologiques et technoéconomiques. Néanmoins, le constat le plus frappant réside dans l'extrême similarité des mesures obtenues pour l'ensemble du Badegoulien récent (de la couche 6 à la couche 21 : fig. 9). Tandis que la proximité des âges obtenus pour les couches 19 à 21 s'accorde bien avec les rapprochements typo-technologiques et taphonomiques effectués pour l'intervalle c.16-21 (fig. 2 : présence de raclettes, de pointes à cran à retouches abruptes et quasiabsence de pointes osseuses ; raccords et remontages lithiques ; Ducasse 2010, p. 30-33, 78 et Ducasse en préparation), leur équivalence avec les mesures disponibles pour les assemblages documentés entre les couches 6 et 15 était moins attendu, compte tenu des différences dont elles témoignent en terme d'équipement cynégétique (quasi disparition des armatures lithiques et développement des pointes osseuses : fig. 2 ; Ducasse et Renard 2012, figure 4 ; Le Guillou 2012). Si tant est que cette " équivalence » radiométrique ne soit pas le fruit de confusions et/ou de perturbations stratigraphiques difficiles

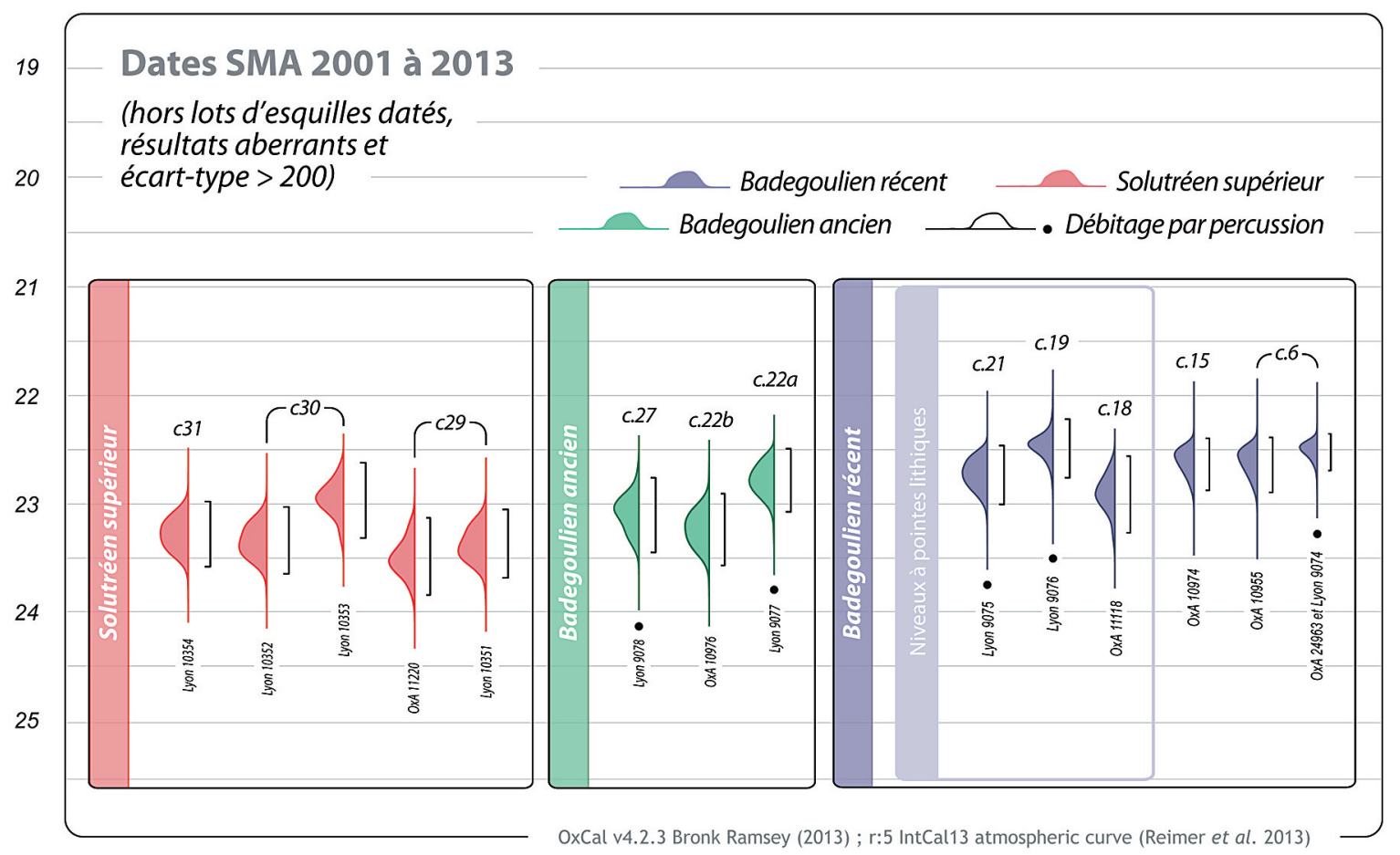

Figure 9 - Synthèse graphique des datations SMA retenues pour la discussion. L'ensemble des calibrations a été réalisé avec la version 4.2.3 du logiciel OxCal (Bronk Ramsey 2013) utilisant la courbe de calibration IntCal13 (Reimer et al. 2013).

Figure 9 - Graphical abstract of the AMS datings selected for discussion. Calibration has been done with the OxCal program (v4.2.3: Bronk Ramsey 2013) using the IntCal13 calibration curve (Reimer et al. 2013). 
à mettre en évidence en l'absence de remontages systématiques, l'apport des données radiométriques s'avère limité, si ce n'est qu'elles nous renseignent sur la fugacité de certains " épisodes " d'occupation et sur la rapidité vraisemblable de certains de ces changements. À l'échelle inter-site, cette distorsion prévisible du temps entre chronologie relative et chronologie ${ }^{14} \mathrm{C}$ contribue probablement à entretenir l'image d'une diversité synchronique de l'armement badegoulien et nous rappelle, si besoin était, que cette « synchronie » n'est souvent qu'une « diachronie courte » (Boëda 2005).

\section{Une démarche à amplifier}

Sans leur accorder un rôle excessif ni oublier leurs limites et écueils, on ne peut que reconnaître l'importance prise par les datations absolues dans le cadre des débats liés aux dynamiques d'évolution des sociétés paléolithiques, que ce soit dans le temps long (rythme des évolutions, corrélations paléo-climatiques et paléo-environnementales) ou dans l'espace (divergences régionales de ces évolutions, mise en place de « mosaïques » culturelles, etc.). De ce point de vue, la " transition » solutréo-badegoulienne se pose comme un terrain d'étude particulièrement fécond. Perçue comme un moment de reconfiguration profonde des normes techno- et socio-économiques (Ducasse 2012 ; Ducasse et Renard 2012), cette « transition » est pourtant mal connue dans ses rythmes, ce qui restreint d'autant la possibilité d'en comprendre les causes précises et d'évaluer plus clairement son éventuelle diversité à l'échelle du sud-ouest de l'Europe. L'état très varié de la documentation archéologique aujourd'hui disponible limite considérablement la portée des hypothèses formulées et exige, zone par zone, une critique rigoureuse.

Si les résultats et interprétations présentés ici se limitent au seul gisement du Cuzoul-de-Vers, cette contribution s'inscrit donc dans une démarche de réévaluation élargie à plusieurs séquences de référence pour le DMG du sudouest de la France (Projet «SaM »: Ducasse et Renard (dir.) ; Morala, Cretin et Ferullo com. orale ${ }^{17}$ ). Gageons que ce renouvellement raisonné des cadres radiométriques, mené conjointement à un travail de critique archéostratigraphique et, autant que faire se peut, à une mise en perspective interdisciplinaire de nos analyses, contribuera prochainement à éclairer certains " angles morts " tout en dessinant des tendances significatives basées sur des données contrôlées et contrôlables.

\section{Remerciements}

II nous est très agréable de remercier ici Jean Clottes et Jean-Pierre Giraud pour la confiance qu'ils nous ont accordée - et nous accordent encore - dans les différentes analyses et réévaluations entreprises autour du Cuzoul-deVers. Nous remercions également très chaleureusement
Pierre Chalard pour son soutien et ses conseils ainsi que le Service Régional de l'Archéologie de Midi-Pyrénées à qui nous devons certaines des datations publiées ici (programme Artémis). Deux des onze mesures ont par ailleurs été financées grâce à une bourse de la Fondation des Treilles obtenue par l'un d'entre nous (JMP). Un très grand merci à Bernard Marty et Olivier Gaiffe qui nous ont facilité l'accès aux industries en bois de cervidé conservées au dépôt régional de Toulouse ainsi qu'à Jean-Christophe Castel pour son aide lors de la sélection des vestiges solutréens. Nous souhaitons également remercier François Lacrampe-Cuyaubère docteur ès Data Desk ${ }^{T M}$ et Olivier Ferullo pour l'accès à certaines données de comparaison. L'obtention de ces données doit ensuite beaucoup à l'aide logistique de Laurent Brou (prêt des trépans) et Didier Galop (accueil dans les locaux du laboratoire GEODE et prêt de petit matériel) que nous remercierons vivement. Merci aux équipes de datation des laboratoires de Lyon (CDRC), de Saclay (CEA) et d'Oxford (ORAU). Toute notre reconnaissance, enfin, à Carolyn Barshay-Szmidt, à Emmanuel Discamps ainsi qu'aux deux relecteurs anonymes dont les commentaires critiques ont permis d'améliorer considérablement la qualité de cet article.

\section{Références bibliographiques}

ALIX C., HARE P.G., ANDREWS T.D., MACKAY G. 2012 A thousand years of lost hunting arrows: wood analysis of ice patch remains in northwestern Canada. Artic, 65, $n^{\circ} 5$, 95-117.

AUJOULAT N., CLEYET-MERLE J.-J., GAUSSEN J., TISNERAT N., VALLADAS H. 1998 - Approche chronologique de quelques grottes ornées paléolithiques du Périgord par datation carbone 14 en spectrométrie de masse par accélérateur de leur mobilier archéologique. Paleo, 10, p. 319-323.

BARANDÍARÁN I. 1988 - Datation C14 de l'art mobilier magdalénien cantabrique. Préhistoire ariégeoise, 43, p. 63-84.

BAUMANN M., MAURY S. 2013 - Ideas no longer written in antler. Journal of Archaeological Science, 40, p. 601-614.

BODU P., DUMARÇAY G., NATON H.-G. 2014 - Un nouveau gisement solutréen en île-de-France, le site des Bossats à Ormesson (Seine-et-Marne). Bulletin de la Société Préhistorique Française, 111, n² 2, p. 225-254.

BOËDA E. 2005 - Paleo-technologie ou anthropologie des techniques? Arob@se, 1, p. 46-64.

BOSSELIN B., DJINDJIAN F. 1988 - Un essai de structuration du Magdalénien français à partir de l'outillage lithique. Bulletin de la Société Préhistorique Française, 85, p. 304-331.

(17) Morala A., Cretin C., Ferullo O. 2011 - De nouveaux jalons chronologiques pour le Badegoulien aquitain : Bordeneuve et Cassegros. Journée d'étude du laboratoire PACEA, "Les sociétés du Dernier Maximum Glaciaire ouest-européen: Badegoulien et Magdalénien", juin 2011, Bordeaux. 
BOSSELIN B., DJINDJIAN F. 1997 - Une révision du solutréen de Laugerie-Haute et le problème des transitions GravettienSolutréen et Solutréen-Badegoulien en Aquitaine. Bulletin de la Société Préhistorique Française, 94, 4, p. 443-454.

BOURDIER C., CHEHMANA L., PÉTILLON J.-M., VALLADAS H. 2014 - L'abri-sous-roche orné de Reverdit (Sergeac, Dordogne) : l'apport d'une approche pluridisciplinaire à l'élaboration d'un nouveau cadre chronoculturel. In : J. Jaubert, N. Fourment et P. Depaepe (dir.), Transitions, ruptures et continuité en Préhistoire, Actes du $27^{\circ}$ Congrès Préhistorique de France, volume 2, Session F: Deuxième moitié et fin du Paléolithique Supérieur. Société Préhistorique Française, Paris, p. 431-447.

BRONK RAMSEY C. 2013 - OxCal Program v4.2., Radiocarbon Accelerator Unit, Université d'Oxford.

BROU L. 2006 - Protocole de datation d'objets manufacturés en matière dure animale. In : D. Sacchi, M. Langlais (Eds.), Projet Collectif de Recherche "Chronologie et Faciès du Magdalénien Méditerranéen ", Rapport Intermédiaire, Service Régional d'Archéologie Languedoc-Roussillon, Montpellier, p. 5-10.

BRYANT C, CARMI I., COOK J. T., GULLIKSEN S., HARKNESS D. D., HEINEMEIER J., MCGEE E., NAYSMITH P., POSSNERT G., SCOTT E. M., Van Der PLICHT J., Van STRYDONCK M. 2001 - Is comparability of ${ }^{14} \mathrm{C}$ dates an issue?: a status report on the Fourth International Radiocarbon Intercomparison, Radiocarbon, $43, n^{\circ} 2$, p. $321-324$

CASTEL J.-C. 1999 - Comportements de subsistance au Solutréen et au Badegoulien d'après les faunes de Combe Saunière (Dordogne) et du Cuzoul-de-Vers (Lot). Thèse de I'Université Bordeaux I, $619 \mathrm{p}$.

CASTEL J.-C. 2003 - Économie de chasse et d'exploitation de l'animal au Cuzoul-de-Vers (Lot) au Solutréen et Badegoulien. Bulletin de la Société Préhistorique Française, 100, $\mathrm{n}^{\circ}$ 1, p. 41-66.

CASTEL J.-C. 2010 - Comportements de subsistance au Solutréen et au Badegoulien d'après les faunes de CombeSaunière (Dordogne) et du Cuzoul-de-Vers (lot), Editions Universitaires Européennes, Sarrebrück, 635 p.

CHAUVIÈRE F.-X., FONTANA L., LANG L., BONANI G., HAJDAS I. 2006 - Une préhampe magdalénienne en bois de renne aux Petits Guinards (Allier, France), C. R. Palevol, 5, p. $725-733$

CHAUVIÈRE F.-X., CASTEL J.-C., L'HOMME X., CAMUS, H., LANGLAIS, M., DAULNY L., DEFOIS B., DUCASSE S., MORALA A., RENARD C., TURQ A. 2008 - Ein neuer Fundort aus dem späten Jungpaläolithikum in Südwestfrankreich: Le Petit Cloup Barrat (Cabrerets, Lot) Quartär, 55, p. 159-163.
CHAUVIÈRE F.-X., BAUMANN M., PÉTILLON J.-M. 2013 - L'industrie osseuse du Grand-Abri de Cabrerets. In : DUCASSE (S.) et RENARD (C.) dir., Sur l'évolution de l'organisation socio-économique des groupes humains entre la fin du Solutréen et les débuts du Magdalénien. Des Causses du Quercy aux contreforts pyrénéens entre 23500 et 18500 cal. BP, rapport de PCR, SRA Midi-Pyrénées, p. 85-92.

CHEHMANA L., DEBOUT G., BODU P. 2013 - Discussion sur l'attribution chronoculturelle de quelques industries à pièces carénées du sud du Bassin parisien. In : P. Bodu, L. Chehmana, L. Klaric, L. Mevel, S. Soriano, N. Teyssandier (dir.), Le Paléolithique supérieur ancien de l'Europe du NordOuest. Réflexions et synthèses à partir d'un projet collectif de recherche sur le centre et le sud du Bassin parisien, Actes du colloque de Sens, 15-18 avril 2009, Société préhistorique française, Mémoire LVI, p.159-171.

CLOTTES, GIRAUD J.-P. 1989 - Le gisement préhistorique du Cuzoul (Vers, Lot), Quercy Recherche, 65-66, p. 82-91.

CLOTTES, GIRAUD J.-P. 2012 - Historique des recherches : la découverte, la fouille et l'étude. In : J. Clottes, J.-P. Giraud, P. Chalard (dir.), Solutréen et Badegoulien au Cuzoul-de-Vers. Des Chasseurs de Rennes en Quercy. ERAUL 131, Liège, p. 461-473.

CLOTTES J., GIRAUD J.-P. et CHALARD P. dir. 2012 Solutréen et Badegoulien au Cuzoul-de-Vers. Des chasseurs de rennes en Quercy (Lot). ERAUL 131, Liège, $488 \mathrm{p}$.

DEBOUT G., OLIVE M., BIGNON O., BODU P., CHEHMANA L., VALENTIN B. 2012 - The Magdalenian in the Paris Basin: New results, Quaternary International, 272-273, p. 176-190.

DELIBRIAS G., GUILLIER M.-T. 1988 - Gif natural radiocarbon measurements $\mathrm{XI}$, Radiocarbon, 30, $\mathrm{n}^{\circ} 1$, p. $61-124$

DETRAIN L., AUBRY T., BEYER C., BIDART P., BOURGUIGNON L., DIOT M.-F., GUADELLI J.-L., KERVAZO B., LEGRAND Y., LEROYER C., LIMONDIN N., MARCON V., MORALA A., PLATEL J.-P., ROUZO P. 1993 - Fouilles préliminaires à l'agrandissement du Musée National de Préhistoire des Eyzies, 1991-1992, AFAN, $84 \mathrm{p}$.

DETRAIN L., AUBRY T., BEYER C., BIDART P., BOURGUIGNON L., DIOT M.-F., GUADELLI J.-L., KERVAZO B., LEGRAND Y., LEROYER C., LIMONDIN N., MARCON V., MORALA A., PLATEL J.-P., ROUZO P. 1994 - Fouilles du Musée National de Préhistoire des Eyzies, Rapport final, AFAN, 84 p. 
DJINDJIAN F. 2000 - Identité, chronologie et territoires du Magdalénien en Europe occidentale : questions posées. In : G. Pion (dir.), Le Paléolithique supérieur récent : nouvelles données sur le peuplement et l'environnement, Actes de la table ronde de Chambéry, Mémoire de la Société Préhistorique Française, 28, p. 95-112.

DUCASSE S. 2010 - La « parenthèse » badegoulienne : fondements et statuts d'une discordance industrielle au travers de l'analyse techno-économique de plusieurs ensembles lithiques méridionaux du Dernier Maximum Glaciaire. Thèse de doctorat, Université de Toulouse-Le Mirail, ex. multigraphié, $460 \mathrm{p}$.

DUCASSE S. 2012 - What is left of the Badegoulian " interlude »? New data on cultural evolution in southern France between 23,500 and 20,500 cal. BP, Quaternary International, 272-273, p. 150-165.

DUCASSE S., RENARD C. avec la collaboration de Bruxelles L., Castel J.-C., Chalard P., Clottes J., Desclaux E., Fourment N., Fritz C., Giraud J.-P., Henri-Gambier D., Kervazo B., Konik S., Le Gall O., Le Guillou Y., Lelouvier L.A., Martin B., Martin H., Morala A., Oberlin C., Pétillon J.-M., Pomiès M.-P., Servelle C., Taborin Y., Turq A., Valladas H., Vignaud C. Et Villotte S. 2012 - De 20000 à 18000 BP en Quercy : apports de la séquence du Cuzoul-de-Vers à la compréhension de l'évolution des comportements socioéconomiques entre Solutréen récent et Badegoulien. In : J. Clottes, J.-P. Giraud, P. Chalard (dir.), Solutréen et Badegoulien au Cuzoul-de-Vers. Des Chasseurs de Rennes en Quercy. ERAUL 131, Liège, p. 461-473.

DUCASSE S., RENARD C. dir. 2013 - Sur l'évolution de l'organisation socio économique des groupes humains entre la fin du Solutréen et les débuts du Magdalénien. Des Causses du Quercy aux contreforts pyrénéens entre 23 500 et $18500 \mathrm{cal}$. BP. Rapport annuel de PCR, Service Régional de l'Archéologie Midi Pyrénées, Toulouse, 194 p.

DUCASSE S., RENARD C. 2014 - Bilan de la campagne de datation 2012/2013. In S. Ducasse et C. Renard (dir.), Projet collectif de recherche «SaM». Du Solutréen au Magdalénien : changements dans l'organisation socioéconomique des groupes humains entre 24 et $19 \mathrm{ka}$ cal. BP. Rapport annuel de PCR, Service Régional de l'Archéologie Midi Pyrénées, Toulouse, p. 23-49.

DUCASSE S., CASTEL J.-C., CHAUVIÈRE F.-X., LANGLAIS M., CAMUS H., MORALA A., TURQ A. 2011 Le Quercy au cœur du Dernier Maximum Glaciaire. La couche 4 du Petit Cloup Barrat et la question de la transition Badegoulo-Magdalénienne, Paleo, 22, p. 101-154.

D'ERRICO F., SANCHEZ GOÑI M.-F., VANHAEREN M. 2006 - L'impact de la variabilité climatique rapide des OIS32 sur le peuplement de l'Europe. In : E. Bard (dir.), L'Homme face au climat, Odile Jacob, Paris, p. 265-282.

EVIN J., MARIEN G., PACHIAUDI Ch. 1976 - Lyon natural radiocarbon measurements $\mathrm{VI}$, Radiocarbon, 18, $\mathrm{n}^{\circ} 1$, p. $60-88$.
EVIN J., MARIEN G., PACHIAUDI Ch. 1978 - Lyon natural radiocarbon measurements VII, Radiocarbon, 20, $\mathrm{n}^{\circ} 1$, p. 19-57.

FOURMENT N. 2002 - La question des sols et niveaux d'habitat du Paléolithique supérieur au Mésolithique : développement d'approches méthodologiques pour l'analyse spatiale de quatre sites entre Massif central et Pyrénées. Thèse de doctorat, Université de Toulouse-Le Mirail, $525 \mathrm{p}$.

FOURMENT N., GIRAUD J.-P. 2012 - Structures d'habitat et organisation de l'espace. In J. Clottes, J.-P. Giraud, P. Chalard (dir.), Solutréen et Badegoulien au Cuzoul-deVers. Des Chasseurs de Rennes en Quercy. ERAUL 131, Liège, p. 407-458.

GENESTE J.-M. 2002 - Éléments pour une contribution intégrée des datations par le radiocarbone aux problématiques de l'archéologie paléolithique. In : Archéologie, patrimoine culturel et datation par le carbone 14 par spectrométrie de masse par accélérateur, Centre de Recherche et de Restauration des Musées de France, le 22 mars 2002, p. 29-34.

HENRY-GAMBIER D., VILLOTTE S. 2012 - Les vestiges humains : deux exemples de traitement du cadavre. In J. Clottes, J.-P. Giraud, P. Chalard (dir.), Solutréen et Badegoulien au Cuzoul-de-Vers. Des Chasseurs de Rennes en Quercy. ERAUL 131, Liège, p. 387-406.

HINGUANT S., BIARD M. 2013 - Le Paléolithique supérieur ancien de la vallée de l'Erve (Mayenne) : un état des connaissances. In : P. Bodu, L. Chehmana, L. Klaric, L. Mevel, S. Soriano, N. Teyssandier (dir.), Le Paléolithique supérieur ancien de l'Europe du Nord-Ouest. Réflexions et synthèses à partir d'un projet collectif de recherche sur le centre et le sud du Bassin parisien, Actes du colloque de Sens, 15-18 avril 2009, Société préhistorique française, Mémoire LVI, Paris, p. 239-250.

KOZLOWSKI S.K., POLTOWICZ-BOBAK M., BOBAK D., TERBERGER T. 2012 - New Information from Maszycka Cave and the Late Glacial Recolonisation of Central Europe, Quaternary International, 272-273, p. 288-296.

LADIER E. 2000 - Le Magdalénien ancien à lamelles a dos de l'abri Gandil a Bruniquel (Tarn-et-Garonne) : étude préliminaire de l'industrie lithique de la C20. In G. Pion (dir.), Le Paléolithique supérieur récent : nouvelles données sur le peuplement et l'environnement, Actes de la table ronde de Chambéry, Mémoire de la Société Préhistorique Française, 28, p. 191-200.

LANGLAIS M. 2010 - Les sociétés magdaléniennes de l'isthme pyrénéen, Documents Préhistoriques 26, Editions du CTHS, Paris, 340 p. 
LANGLAIS M., PÉTILLON J.-M., DUCASSE S., LENOIR M. 2010 - Badegoulien versus Magdalénien. Entre choc culturel et lente transition dans l'Aquitaine paléolithique. In : V. Mistrot (coord.), De Néandertal à l'Homme Moderne. L'Aquitaine préhistorique, vingt ans de découvertes (19902010), Éd. Confluences, Bordeaux, p.116-129.

LE GUILLOU Y. 2012 - Premier regard sur la matière dure animale ouvragée. In : J. Clottes, J.-P. Giraud, P. Chalard (dir.), Solutréen et Badegoulien au Cuzoul-de-Vers. Des Chasseurs de Rennes en Quercy. ERAUL 131, Liège, p. 279-354.

LEMOZI A. 1961 - Le Grand Abri sous roche solutréen de Cabrerets. Bulletin de la Société des Etudes Littéraires, Scientifiques et Artistiques du Lot, 82, p. 100-106.

MARGO Project Members 2009 - Contraints on the magnitude and patterns of ocean cooling at the Last Glacial Maximum, Nature Geoscience, 2, 127-132.

MIX A., BARD E., SCHNEIDER R. 2001 - Environemental processes of the ice age: land, oceans, glaciers (EPILOG), Quaternary Science Reviews, 20, p. 627-657.

OBERLIN Ch., VALLADAS H. 2012 - Le cadre chronologique : datation ${ }^{14} \mathrm{C}$. In : J. Clottes, J.-P. Giraud, P. Chalard (dir.), Solutréen et Badegoulien au Cuzoul-deVers. Des Chasseurs de Rennes en Quercy. ERAUL 131, Liège, p. 79-84.

PÉTILLON J.-M., AVERBOUH A. 2012 - Le travail du bois de renne dans les couches badegouliennes. In : J. Clottes, J.-P. Giraud, P. Chalard (dir.), Solutréen et Badegoulien au Cuzoul-de-Vers. Des Chasseurs de Rennes en Quercy. ERAUL 131, Liège, p. 363-390.

PÉTILLON J.-M., DUCASSE S. 2012 - From flakes to grooves: a technical shift in antlerworking during the last glacial maximum in southwest France, Journal of Human Evolution, 62, p. 435-465.

PÉTILLON J.-M., LANGLAIS M., KUNTZ D., NORMAND C., BARSHAY-SZMIDT C., COSTAMAGNO S., DELMAS M., LAROULANDIE V., MARSAN G. sous presse - The human occupation of the northwestern Pyrenees in the Late Glacial: new data from the Arudy basin, lower Ossau valley. Quaternary International.

PRIMAULT J., BROU L., GABILLEAU J. et LANGLAIS M. 2007 - La grotte du Taillis des Coteaux à Antigny (Vienne) : intérêts d'une séquence originale à la structuration des premiers temps du Magdalénien. Bulletin de la Société Préhistorique Française, 104, n² 4, p. 743-758.

REIMER P.J., BARD E., BAYLISS A., BECK J.W., BLACKWELL P.G., BRONK RAMSEY C., BUCK C.E., CHENG H., EDWARDS R.L., FRIEDRICH M., GROOTES P.M., GUILDERSON T.P., HAFLIDASON H., HAJDAS I., HATTE C., HEATON T.J., HOFFMANN D. L., HOGG A.G., HUGHEN K.A., KAISER K.F., KROMER B., MANNING S.W., NIU M., REIMER R.W., RICHARDS D.A.,
SOUTHON J.R., STAFF R. A., TURNEY C.S.M., PLICHT J. van der 2013 - Intcal13 and marine13 Radiocarbon Age Calibration Curves, 0-50,000 Years cal BP, Radiocarbon, 55,4 , p. 1869-1887.

RENARD C. 2008 - Les premières expressions du Solutréen dans le Sud-Ouest français. Evolution technoéconomique des équipements lithiques au cours du dernier maximum glaciaire. Thèse de doctorat, Université de Nanterre-Paris X, 449 p.

RENARD C. 2012 - Les industries lithiques du Solutréen : l'organisation des productions en silex, implications technoéconomiques. In : J. Clottes, J.-P. Giraud, P. Chalard (dir.), Solutréen et Badegoulien au Cuzoul-de-Vers. Des Chasseurs de Rennes en Quercy. ERAUL 131, Liège, p. 101-132.

ROQUE C., GUIBERT P., VARTANIAN E., BECHTEL F., OBERLIN C., EVIN J., MERCIER N., VALLADAS H., TEXIER J.-P., RIGAUD J.-PH., DELPECH F., CLEYETMERLE J.-J., TURQ A. 2001 - Une expérience de croisement de datations $\mathrm{TL} / 14 \mathrm{C}$ pour la séquence solutréenne de Laugerie-Haute, Dordogne, In : Barrandon J.N., Guibert P., Michel V. (éds) Datation, XXle Rencontres Internationales d'Archéologie et d'Histoire d'Antibes, Editions APDCA, Antibes, p. 217-232.

SACCHI D. 2003 - Retour sur la station Badegoulienne de Lassac (Aude), Bulletin de la Société Préhistorique AriègePyrénées, 58, p. 157-162.

SACCHI D., BRULÉ J.-L., DUCASSE S. (Ed.) à paraître La station badegoulienne de Lassac à Sallèles-Cabardès (Aude) : le Locus 1. GAEP, Carcassonne.

SCOTT E. M. 2003 - The Third International Radiocarbon Intercomparison (TIRI) and the Fourth International Radiocarbon Intercomparison (FIRI), Radiocarbon, 45, $\mathrm{n}^{\circ} 2$, p. $135-328$

SCOTT E. M., COOK G. T., NAYSMITH P., BRYANT C., O'DONNELL D. 2007 - A report on phase 1 of the 5th International Radiocarbon Intercomparison (VIRI), Radiocarbon, 49, $\mathrm{n}^{\circ}$ 2, p. 409-426.

SCOTT E. M., COOK G. T., NAYSMITH P. 2010a - A report on phase 2 of the 5th International Radiocarbon Intercomparison (VIRI), Radiocarbon, 52, $\mathrm{n}^{\circ} 2-3$, p. 846-858.

SCOTT E. M., COOK G. T., NAYSMITH P. 2010b - The Fifth International Radiocarbon Intercomparison (VIRI): an assessment of laboratory performance in stage 3, Radiocarbon, 52, $\mathrm{n}^{\circ}$ 2-3, p. 859-865.

SEDLMEIER J. 2010 - Die jungpalaolithischen Funde aus der Mittleren Fundschicht der Kastelhöhle Nord im nordwestschweizerischen Kaltbrunnental (Gemeinde Himmelried SO). Ein Nachweis für die Begehung der Schweiz durch Mensch und Tier kurz nach dem letzten Kaltemaximum der letzten Eiszeit, Jahrbuch Archäologie der Schweiz, 93, p. 7-34. 
SZMIDT C., PÉTILLON J.-M., CATTELAIN P., NORMAND C., SCHWAB C., 2009 - Premières dates radiocarbone pour le Magdalénien d'Isturitz (Pyrénées-Atlantiques). Bulletin de la société préhistorique française, 106, p. 588-592.

TERBERGER T. 2013 - Le Dernier Maximum glaciaire entre le Rhin et le Danube, un réexamen critique. In $\mathrm{P}$. Bodu, L. Chehmana, L. Klaric, L. Mevel, S. Soriano, N. Teyssandier (dir.), Le Paléolithique supérieur ancien de l'Europe du Nord-Ouest. Réflexions et synthèses à partir d'un projet collectif de recherche sur le centre et le sud du Bassin parisien, Actes du colloque de Sens, 15-18 avril 2009, Société préhistorique française, Mémoire LVI, Paris, p.415-443.

TISNERAT-LABORDE N., VALLADAS H., LADIER E. 1997 - Nouvelles datations carbone 14 en SMA pour le Magdalénien supérieur de la vallée de l'Aveyron. Préhistoire ariégeoise, 52, p. 129-136.
VALLADAS H. 2002 - La calibration des âges carbone 14 pour le Paléolithique supérieur. In : Archéologie, patrimoine culturel et datation par le carbone 14 par spectrométrie de masse par accélérateur, Centre de Recherche et de Restauration des Musées de France, le 22 mars 2002, p. 44-47.

VALLADAS H., TISNERAT-LABORDE N., OBERLIN Ch. 2001 - Datation carbone 14 en spectométrie de masse par accélérateur et archéologie. In J.-N. Barrandon, P. Guibert, V. Michel (dir.), Datation. Actes des $\mathrm{XXI}^{\mathrm{e}}$ rencontres internationales d'archéologie et d'histoire d'Antibes, 19-21 octobre 2000, Editions APDCA, Antibes, p. 81-91. 